\title{
THE HEIGHT OF INCREASING TREES
}

\author{
MICHAEL DRMOTA
}

ABSTRACT. Increasing trees have been introduced by Bergeron, Flajolet and Salvy [1]. This kind of notion covers several well knows classes of random trees like binary search trees, recursive trees, and plane oriented (or heap ordered) trees.

We consider the height of increasing trees and prove for several classes of trees (including the above mentioned ones) that the height satisfies $\mathbf{E} H_{n} \sim$ $\gamma \log n$ (for some constant $\gamma>0$ ) and $\operatorname{Var} H_{n}=O(1)$ as $n \rightarrow \infty$. The methods uses are based on generating functions.

\section{INTRODUCTION}

Increasing trees have been introduced by Bergeron, Flajolet and Salvy [1]. They constitute classes of weighted planar or non-planar recursive trees.

A recursive tree is a (planar or non-planar) rooted tree (witn $n$ nodes) where the nodes are labeled with $1,2, \ldots, n$ such that all successors of each node have a larger label. In particular, the root has label 1, and every path from the root to a leaf has strictly increasing labels. It is also possible to consider a recursive tree as the result of an evolution process. The process starts with the root (that gets label 1 ). Next, another node is attachted to the root (that gets label 2) and in every step a new node is attachted to an already existing node (and gets the next label). The labels are the history of the tree evolution.

Now we consider the class $J$ of all planar (resp. non-planar) recursive trees and associate a weight to these trees in the following way. Let $\psi_{j}, j \geq 0$, be a given sequence of non-negative numbers with $\psi_{0}>0$. Then the weight $\omega(t)$ of a recursive tree $t$ is defined by

$$
\omega(t)=\prod_{j \geq 0} \psi_{j}^{D_{j}(t)},
$$

where $D_{j}(t)$ denotes the number of nodes in $t$ with $j$ successors. Let $J_{n}$ denote the set of recursive trees of size $n$ then we set

$$
y_{n}=\sum_{t \in J_{n}} \omega(t)
$$

and

$$
y(z)=\sum_{n \geq 0} y_{n} \frac{z^{n}}{n !} .
$$

By definition it is clear that the generating function $y(z)$ satisfies the differential equation

$$
y^{\prime}(z)=\Psi(y(z)), \quad y(0)=0
$$

Date: December 8, 2006.

Institut für Diskrete Mathematik und Geometrie, TU Wien, Wiedner Hauptstrasse 8-10/118, A-1040 Wien, Austria, email: michael.drmota@tuwien.ac.at.

This research was supported by the Austrian Science Foundation FWF, project S9604, that is part of the Austrian National Research Network "Analytic Combinatorics and Probabilistic Number Theory". 
where

$$
\Psi(w)=\sum_{j \geq 0} \psi_{j} w^{j}
$$

if we consider planar recursive trees and

$$
\Psi(w)=\sum_{j \geq 0} \psi_{j} \frac{w^{j}}{j !}
$$

if we consider non-planar recursive trees. Note that the set of non-planar trees can be obtained from planar trees by considering the natural equivalence relation. Thus, we will mainly focus on the planar version.

Before we state some known results on increasing trees we want to recall the most prominent examples of increasing trees.

Example . Binary increasing trees are defined by $\Psi(w)=(1+w)^{2}$. We have $y_{n}=n$ ! and $y(z)=1 /(1-z)$. The probabilty model that is induced by this (planar) binary increasing trees (see below) is exactly the standard permutation model of binary search trees.

Example . Recursive trees (that is, every non-planar recursive tree gets label 1) are given by $\Psi(w)=e^{w}$. Here $y_{n}=(n-1)$ ! and $y(z)=\log (1 /(1-z))$.

Example . Plane oriented (recursive) trees (or heap ordered trees) are given by $\Psi(w)=1 /(1-w)$. This means that every planar recursive trees gets label 1 . Here $y_{n}=(2 n-3) ! !=1 \cdot 3 \cdot 5 \cdots(2 n-3)$ and $y(z)=1-\sqrt{1-2 z}$.

The papers of Bergeron, Flajolet and Salvy [1] and Smythe and Mahmoud [15] give a good survey on known results on theses kinds of trees, in particular on recursive and plane oriented trees.

The reason for introducing weights is that they induce a natural probability model on $J_{n}$. The probability of a tree $t \in J_{n}$ is given by $P_{n}(t)=\omega(t) / y_{n}$. Every parameter on increasing trees is then a random variable and it is a natural problem to study the asymptotic distribution of these random variables. (In this paper we will focus on the height $H_{n}$ of increasing trees of size $n$.) Bergeron, Flajolet and Salvy [1] have already studied several parameters, for example the depth. More precisely, let $\Omega_{n}$ denote the typical depth of a node in $J_{n}$, that is, the depth of a random node (where every node in equally likely) in a random tree in $J_{n}$. The probability distrbution of $\Omega_{n}$ is given by

$$
P\left(\Omega_{n}=k\right)=\frac{\mathbf{E} L_{n, k}}{n},
$$

where $L_{n, k}$ denotes the number of nodes at level $k$. (The sequence $\left(L_{n, k}\right)_{k \geq 0}$ is also called profile of trees in $J_{n}$ and desribes more of less the shape of a random incresing tree.) In [1] it is shown that $\Omega_{n}$ satisfies a central limit theorem of the form

$$
\frac{\Omega_{n}-C \log n}{\sqrt{C \log n}} \rightarrow N(0,1)
$$

where $C=\frac{d}{d-1}$ if $\Psi(w)$ is a polynomial of degree $d, C=1$ for recursive trees, and $C=\frac{1}{2}$ for plane oriented trees.

Since the distribution of $\Omega_{n}$ is closely related to the expected profile $\mathbf{E} L_{n, k}$ this central limit theorem gives an overall picture of the shape of increasing trees. They are so-called $\log n$-trees, that is, the average depth of nodes in trees of size $n$ is of order $\log n$.

The purpose of this paper is to make the statement of $\log n$-tree more accurate in the sense that we will consider the distribution of the height $H_{n}$ and show that $H_{n}$ is also of order $\log n$. We will also observe a phenomenon that occurs quite frequently for $\log n$-trees, namely that the distribution of the height is highly concentrated, in particular the variance $\operatorname{Var} H_{n}$ is bounded as $n \rightarrow \infty$. 
There are not many results on the height of increasing trees that are available in the literature. Of course, the height binary search trees has been well studied, see $[4,5,6,8,9,14]$. Further, Pittel [13] has already considered recursive trees $\left(\Psi(w)=e^{w}\right)$ and scale-free trees $\left(\Psi(w)=(1-w)^{-r}\right)$ and has proved that

$$
\frac{H_{n}}{\log n} \rightarrow c \quad \text { in probability }
$$

for proper height constants $c>0$. These two kind of trees will be discussed in detail in Theorems 3 and 4. Finally, Broutin et al. [2] proved a similar relation for increasing trees that are defined by polynomials $\Psi(w)$. Interestingly the height constant $c$ depends only on the degree $d$ of $\Psi(w)$. In Theorems 1 and 2 we will also consider these kinds of trees, however, we will only get precise results for $d$-ary increasing trees defined by $\Psi(w)=(1+w)^{d}$.

\section{Results}

Let $y(z)=\sum_{n \geq 0} y_{n} z^{n} / n$ ! the generating function of a class of increasing trees and let $H_{n}$ denote the (random) height of increasing trees of size $n$. Then we introduce the generating functions

$$
y_{k}(z)=\sum_{n \geq 0} y_{n} \mathbf{P}\left\{H_{n} \leq k\right\} \frac{z^{n}}{n !} .
$$

By recalling the recursive description of increasing trees it easily follows that $y_{0}(z)=$ 0 and

with $y_{k+1}(0)=0$.

$$
y_{k+1}^{\prime}(z)=\Psi\left(y_{k}(z)\right)
$$

Theorem 1. Suppose that $\Psi(w)$ is an aperiodic polynomial, that is, $\Psi(w)$ cannot be written in the form $\Psi(w)=\Phi\left(w^{r}\right)$ for some $r>1$ and some polynomial $\Phi(w)$, and let $\rho$ denote the radius of convergence of $y(z)$.

Then we have, as $n \rightarrow \infty$,

$$
\mathbf{E} H_{n}=O(\log n) .
$$

Furthermore, if $\mathbf{P}\left\{H_{n+1} \leq k\right\} \leq \mathbf{P}\left\{H_{n} \leq k\right\}$ for all $n$ and $k$ and if there exists $\delta>0$ such that $y_{k+1}(\rho) \geq(1+\delta) y_{k}(\rho)$ (for all $k \geq 0$ ) then we have, as $n \rightarrow \infty$,

$$
\operatorname{Var} H_{n}=O(1)
$$

and exponential tail estimates of the form

$$
\mathbf{P}\left\{\left|H_{n}-\mathbf{E} H_{n}\right| \geq \eta\right\} \ll e^{-c \eta}
$$

for some $c>0$.

Remark . Unfortunately we cannot show the conditions $\mathbf{P}\left\{H_{n+1} \leq k\right\} \leq \mathbf{P}\left\{H_{n} \leq\right.$ $k\}$ and $y_{k+1}(\rho) \geq(1+\delta) y_{k}(\rho)$ in general. We conjecture that both conditions are satisfied if the sequence $\psi_{j}$ is log-concave, that is, $\psi_{j-1} \psi_{j+1} \leq \psi_{j}^{2}$. Concerning the second condition we mention that it is quite easy to show (see Lemma 7) that $y_{k}(\rho) \gg$ $(1+\delta)^{k}$ for some $\delta>0$. Thus, it is very likely that it is actually satisfied for all polynomial increasing trees. Furthermore, if we also know that $y_{k+1}(\rho) / y_{k+1}(\rho) \rightarrow \alpha>1$ as $k \rightarrow \infty$ then it also follows that $\mathbf{E} H_{n} \sim(1 / \log \alpha) \log n$.

For special polynomials we can be more precise. The following theorem covers the case of so-called $d$-ary increasing trees.

Theorem 2. Suppose that $\Psi(w)=\psi_{0}\left(1+\psi_{1} /\left(d \psi_{0}\right) w\right)^{d}$ with positive real numbers $\psi_{0}, \psi_{1}$ and an integer $d \geq 2$.

Then, as $n \rightarrow \infty$, we have

$$
\mathbf{E} H_{n}=c_{d} \log n+O(\sqrt{\log n}(\log \log n))
$$


where $c_{d}$ is the largest positive real solution of the equation

$$
c_{d} \log \frac{d e}{c_{d}(d-1)}=\frac{1}{d-1} .
$$

Furthermore we have (uniformly for all $k \geq 0$ as $n \rightarrow \infty$ )

$$
\mathbf{P}\left\{H_{n} \leq k\right\}=F\left((d-1) n / y_{k}(\rho)^{d-1}\right)+o(1),
$$

where $\rho=d /\left((d-1) \psi_{1}\right)$ and $F(y)$ satisfies the integral equation

$$
y^{\frac{1}{d-1}} F\left(y e^{-1 / c_{d}}\right)=\frac{\Gamma\left(\frac{d}{d-1}\right)}{\Gamma\left(\frac{1}{d-1}\right)^{d}} \int_{y_{1}+\cdots+y_{d}=y, y_{j} \geq 0} \prod_{j=1}^{d}\left(F\left(y_{j}\right) y_{j}^{\frac{1}{d-1}-1}\right) d \mathbf{y}
$$

Moreover, as $n \rightarrow \infty$,

$$
\operatorname{Var} H_{n}=O(1)
$$

and there are exponential tail estimates of the form

$$
\mathbf{P}\left\{\left|H_{n}-\mathbf{E} H_{n}\right| \geq \eta\right\} \ll e^{-c \eta}
$$

with some $c>0$

Remark . The limit relation (2) can be interpreted in the following way. Let $h_{n}$ be defined by $h_{n}=\max \left\{k: y_{k}(\rho)^{d-1} \leq n\right\}$ and set $W(x)=F\left(e^{-x}\right)$. Then

$$
\mathbf{P}\left\{H_{n} \leq h_{n}+r\right\}=W\left(\log \frac{y_{h_{n}}(\rho)^{d-1}}{(d-1) n}+\frac{r}{c_{d}}\right)+o(1) .
$$

Here we have also used the relation $y_{k+1}(\rho) / y_{k}(\rho) \sim e^{1 /\left(c_{d}(d-1)\right)}$ (see Lemma 9$)$ and continuity of $F$. Thus, the probability distribution of $H_{n}$ around $h_{n}$ is almost the same for all $n$. There is only a fluctuating shift that has its origin in the discretization level of the problem, both, $n$ and $k$ are discrete. (Note that $e^{-1 / c_{d}} \leq y_{h_{n}}(\rho)^{d-1} / n \leq$ $e^{1 / c_{d}}$.)

The function $W(x)$ can be seen as a travelling wave (compare also with [3]).

Remark. We want to note that for the binary case $\Psi(w)=(1+w)^{2}$ (that is equivalent to binary search trees) various results of that kind (and even much more precise ones) are well known, see [4, 9, 14]. For example, in the binary case one has

$$
\mathbf{E} H_{n}=c_{2} \log n-\frac{3 c_{2}}{2\left(c_{2}-1\right)} \log \log n+O(1) \text {. }
$$

Of course, we expect similar relations for all polynomial classes of increasing trees.

The next two theorems cover recursive trees and scale-free trees.

Theorem 3. Suppose that $\Psi(w)=\psi_{0} e^{\frac{\psi_{1}}{\psi_{0}} w}$ with $\psi_{0}>0, \psi_{1}>0$. Then

$$
\mathbf{E} H_{n}=e \log n+O(\sqrt{\log n}(\log \log n)) \text {. }
$$

Furthermore we have (uniformly for all $k \geq 0$ as $n \rightarrow \infty$ )

$$
\mathbf{P}\left\{H_{n} \leq k\right\}=F\left(n / y_{k}^{\prime}(\rho)\right)+o(1),
$$

where $\rho=1 / \psi_{1}$ and $F(y)$ satisfies the integral equation

$$
y F\left(y / e^{1 / e}\right)=\int_{0}^{y} F\left(z / e^{1 / e}\right) F(y-z) d z .
$$

Moreover, as $n \rightarrow \infty$,

$$
\operatorname{Var} H_{n}=O(1)
$$

and there are exponential tail estimates of the form

$$
\mathbf{P}\left\{\left|H_{n}-\mathbf{E} H_{n}\right| \geq \eta\right\} \ll e^{-c \eta}
$$

with some $c>0$ 
Theorem 4. Suppose that $\Psi(w)=\psi_{0}\left(1-\frac{\psi_{1}}{r \phi_{0}} w\right)^{-r}$ for some rational number $r=\frac{A}{B}>0$ (with positive coprime integers $\left.A, B\right)$. Set $c_{r}^{\prime}=r /((r+1) \gamma$ ), where $\gamma$ is the real solution of $\gamma e^{1+\gamma / r}=1$ Then

$$
\mathbf{E} H_{n} \sim c_{r}^{\prime} \log n .
$$

Furthermore we have (uniformly for all $k \geq 0$ as $n \rightarrow \infty$ )

$$
\mathbf{P}\left\{H_{n} \leq k\right\}=G\left((r+1) n /\left(y_{k}^{\prime}(\rho)\right)^{1+\frac{1}{r}}\right)+o(1),
$$

where $\rho=r /\left((r+1) \psi_{1}\right)$. $G(y)$ is given by

$$
G(y)=\frac{\Gamma\left(\frac{A}{A+B}\right)}{\Gamma\left(\frac{1}{A+B}\right)^{A}} \int_{z_{1}+\cdots+z_{A}=1, z_{j} \geq 0} \prod_{j=1}^{A}\left(F\left(y z_{j}\right) z_{j}^{\frac{1}{A+B}-1}\right) d \mathbf{z}
$$

and $F(y)$ satisfies the integral equation

$$
\begin{aligned}
y^{\frac{1}{A+B}} F\left(y e^{-1 / c_{r}^{\prime}}\right)=\frac{\Gamma\left(1+\frac{1}{A+B}\right)}{\Gamma\left(\frac{1}{A+B}\right)^{A+B+1}} \int_{y_{1}+\cdots+y_{A+B+1}=y, y_{j} \geq 0} & \prod_{j=1}^{B+1}\left(F\left(y_{j} e^{-1 / c_{r}^{\prime}}\right) y_{j}^{\frac{1}{A+B}-1}\right) \\
& \times \prod_{\ell=B+2}^{A+B+1}\left(F\left(y_{\ell}\right) y_{\ell}^{\frac{1}{A+B}-1}\right) d \mathbf{y} .
\end{aligned}
$$

Moreover, as $n \rightarrow \infty$,

$$
\operatorname{Var} H_{n}=O(1)
$$

and there are exponential tail estimates of the form

$$
\mathbf{P}\left\{\left|H_{n}-\mathbf{E} H_{n}\right| \geq \eta\right\} \ll e^{-c \eta}
$$

with some $c>0$.

Remark. There is no doubt that Theorem 4 has an analogue for irrational $r>0$. However, the methods of this paper are not strong enough to prove a relation of the form (5) for irrational $r$. Nevertheless, exponential bounds can be shown for all cases, compare with [10].

The paper is organized as follows. In Section 3 we present an alternate description of the underlying probabilistic model that is based on an evolution model and works for $d$-ary increasing trees, for recursive trees and scale-free trees. In Sections 4-7 we prove Theorems 1-4 separately.

\section{The Probabilistic Model}

As mentioned above, (non-planar) recursive trees can be described by a tree evolution process. We start with one node (with label 1) and at each step we attach randomly a new node to one of the preceding ones. It is clear that every recursive tree of size $n$ is generated with equal probability $1 /(n-1)$ !. In other words, this tree evolution process induces uniform distribution on recursive trees of size $n$. Similarly this works for binary increasing trees and also for plane oriented trees.

In a recent paper Panholzer and Prodinger [12] proved that there are exactly three families where the sequence $P_{n}$ of probability measures on $J_{n}$ is induced by a (natural) tree evolution process (described below) if and only if $\Psi(t)$ has one of the three forms:

- $\Psi(w)=\psi_{0}\left(1+\left(\psi_{1} /\left(d \psi_{0}\right)\right) w\right)^{d}$ for some $d \in\{2,3, \ldots\}$ and $\psi_{0}>0, \psi_{1}>0$.

- $\Psi(w)=\psi_{0} e^{\frac{\psi_{1}}{\psi_{0}} w}$ with $\psi_{0}>0, \psi_{1}>0$.

- $\Psi(w)=\psi_{0}\left(1-\frac{\psi_{1}}{r \phi_{0}} w\right)^{-r}$ for some $r>0$ and $\psi_{0}>0, \psi_{1}>0$ 
The corresponding tree evolution process runs as follows ${ }^{1}$ The starting point is (again) one node (the root) with label 1 . Now assume that a tree $t$ is size $n$ is present. We attach to every node $v$ of $t$ a local weight $\rho(v)=(k+1) \psi_{k+1} \psi_{0} / \psi_{k}$ when $v$ has $k$ successors and set $\rho(t)=\sum_{v \in t} \rho(v)$. Observe that in a planar tree there are $k+1$ differnt ways to attack a new (labeled) node to an (already existing) node with $k$ successors. Now choose a node $v$ in $t$ according to the probability distrbution $\rho(v) / \rho(t)$ and and then independently and uniform one of the $k+1$ possibilities to attach a new node there (when $v$ has $k$ successors). This construction ensures that in these three particular cases a tree $t$ of size $n$ that occurs with probability proportional to $\omega(t)$ generates a tree $t^{\prime}$ of size $n+1$ with probability that is proportional to $\omega(t) \psi_{k+1} \psi_{0} / \psi_{k}$ which equals $\omega\left(t^{\prime}\right)$. Thus, this procedure induces the same probability distribution on $J_{n}$ as the above mentionen one where a tree $t \in J_{n}$ has probablility $\omega(t) / y_{n}$.

Note that if we are only interesed in the distributions $P_{n}$ then we can work (without loss of generality) with some special values for $\psi_{0}$ and $\psi_{1}$. It is sufficient to consider the generating functions

- $\Psi(w)=(1+w)^{d}$ for some $d \in\{2,3, \ldots\}$ ( $d$-ary increasing trees).

- $\Psi(w)=e^{w}$ (recursive trees).

- $\Psi(w)=(1-w)^{-r}$ for some $r>0$ (generalized plane oriented trees).

In the third class, the probabilty of choosing a node with out-degree $j$ is proportional to $j+r$.

This tree evolution model can be used in several ways. For example one can ask whether there are properties that hold almost surely. On the other hand several relations are automatically true. For example in the framework of the tree evolution we definitely have $H_{n+1} \geq H_{n}$ and consequently

$$
\mathbf{P}\left\{H_{n+1} \leq k\right\} \leq \mathbf{P}\left\{H_{n} \leq k\right\} .
$$

When one only considers the probability distributions on $J_{n}$ resp. on $J_{n+1}$ this relation is far from beeing obvious and, in fact, is not true in all cases. However, we conjecture that it is true if the sequence $\psi_{j}$ is log-concave. This conjecture is supported by the oberervation that (6) can be directly checked for small $n$ and $k$ under the log-concavity assumption.

\section{Polynomial Increasing Trees}

In this section we will prove Theorem 1. First we recall a property for the singular behaviour of $y(z)$, see [1].

Lemma 1. Suppose that $\Psi(w)$ is an aperiodic polynomial of degree $d$ with leading coefficient $\psi_{d}$. Then

$$
\rho=\int_{0}^{\infty} \frac{d y}{\Psi(y)}
$$

is the only dominant singularity of $y(z)$.

Furthermore, there exists an analytic function $H(w)=\sum_{m \geq 0} h_{m} w^{m}$ with $h_{0}=1$ such that

$$
y(z)=\frac{1}{\eta\left(1-\frac{z}{\rho}\right)^{1 /(d-1)}} H\left(\eta\left(1-\frac{z}{\rho}\right)^{1 /(d-1)}\right)
$$

locally around $z=\rho$, where $\eta=\left((d-1) \rho \psi_{d}\right)^{1 /(d-1)}$. Consequently,

$$
y_{n} \sim n ! \frac{\rho^{-n}}{\eta} \frac{n^{\frac{2-d}{d-1}}}{\Gamma\left(\frac{1}{d-1}\right)} .
$$

\footnotetext{
${ }^{1}$ For the sake of brevity we only discuss the planar version.
} 
For example, if $\Psi(w)=(1+w)^{d}$ then $\rho=1 /(d-1)$,

$$
y(z)=(1-(d-1) z)^{\frac{1}{d-1}}-1
$$

and

$$
y_{n}=n !(-(d-1))^{n}\left(\begin{array}{c}
-\frac{1}{d-1} \\
n
\end{array}\right) \sim n !(d-1)^{n} \frac{n^{\frac{2-d}{d-1}}}{\Gamma\left(\frac{1}{d-1}\right)} .
$$

For the next lemma we use the notation $A(z) \leq_{c} B(z)$ which means that all coefficients of the power series $A(z)$ are smaller or equal than the corresponding coefficients of $B(z)$. Of course, $A(z) \leq_{c} B(z)$ implies $A(z) \leq B(z)$ for all $z \geq 0$.

Lemma 2. Set $\delta_{k}(z)=y(z)-y_{k}(z)$. Then there exists a constant $C>0$ such that

$$
\delta_{k}(z) \leq_{c} C \sum_{\ell \geq k} \frac{1}{\ell !}(\log \Psi(y(z)))^{\ell} .
$$

Proof. We proceed by induction. Note that $y(z) \leq_{c} C y^{\prime}(z)$ (for some constant $C>0$ ) and thus

$$
\delta_{0}(z)=y(z) \leq_{c} C y^{\prime}(z)=C \Psi(y(z))=C \sum_{\ell \geq 0} \frac{1}{\ell !}\left(\log \Psi(y(z))^{\ell} .\right.
$$

Next observe that

$$
\begin{aligned}
\left(\sum_{\ell \geq k+1} \frac{1}{\ell !}(\log \Psi(y(z)))^{\ell}\right)^{\prime} & =\sum_{\ell \geq k+1} \frac{1}{(\ell-1) !}(\log \Psi(y(z)))^{\ell-1} \frac{\Psi^{\prime}(y(z))}{\phi(y(z))} y^{\prime}(z) \\
& =\sum_{\ell \geq k} \frac{1}{\ell !}(\log \Psi(y(z)))^{\ell} \Psi^{\prime}(y(z)) .
\end{aligned}
$$

Consequently,

$$
\begin{aligned}
\delta_{k+1}^{\prime}(z) & =\Psi(y(z))-\Psi\left(y_{k}(z)\right) \\
& =\Psi^{\prime}(\xi(z)) \delta_{k}(z) \\
& \leq{ }_{c} \Psi^{\prime}(y(z)) \delta_{k}(z) \\
& =\Psi^{\prime}(y(z)) \sum_{\ell \geq k} \frac{1}{\ell !}(\log \Psi(y(z)))^{\ell} \\
& =\left(\sum_{\ell \geq k+1} \frac{1}{\ell !}(\log \Psi(y(z)))^{\ell}\right)^{\prime} .
\end{aligned}
$$

Finally, by integration both sides from 0 to $z$ we have completed the inductive proof.

Lemma 3. Suppose that $\Psi(w)$ is a polynomial of degree $d$ and that $k \geq(1+$ $\varepsilon) \frac{d-1}{d} \log n$ for some $\varepsilon>0$. Then

$$
\left[z^{n}\right] \delta_{k}(z) \ll \frac{\rho^{-n}}{n k !}\left(\frac{d}{d-1} \log n\right)^{k}
$$

Proof. Lemma 1 implies that $\log \Psi(y(z)) \sim \frac{d}{d-1} \log (1 /(1-z / \rho))$. Hence

$$
\left[z^{n}\right] \log \Psi(y(z))^{\ell} \ll \frac{\rho^{-n}}{n}\left(\frac{d}{d-1} \log n\right)^{\ell} .
$$

follows from the methods used for the proof of [8, Lemma 4.2].

Now, (7) is an easy consequence of Lemma 2 and (8). 
Lemma 4. Suppose that $\Psi(w)$ is a polynomial of degree $d$. Then for all $\gamma \geq(1+$ $\varepsilon) \frac{d-1}{d}$ we have

$$
\mathbf{P}\left\{H_{n}>\gamma \log n\right\} \ll n^{\gamma-\frac{1}{d-1}+\gamma \log \frac{d}{d+1}-\gamma \log \gamma}
$$

and consequently

$$
\mathbf{E} H_{n} \leq \tilde{\gamma} \log n+O(1),
$$

where $\tilde{\gamma}>0$ is the largest solution of

$$
\tilde{\gamma}-\frac{1}{d-1}+\tilde{\gamma} \log \frac{d}{d+1}-\tilde{\gamma} \log \tilde{\gamma}=0
$$

Proof. Let $k=\gamma \log n \geq(1+\varepsilon) \frac{d-1}{d} \log n$. Then

$$
\begin{aligned}
\mathbf{P}\left\{H_{n}>k\right\} & =\frac{n !}{y_{n}}\left[z^{n}\right] \delta_{k}(z) \\
& \ll \frac{n !}{y_{n}} \frac{\rho^{-n}}{n k !}\left(\frac{d}{d-1} \log n\right)^{k} \\
& \ll n^{-\frac{1}{d-1}+\gamma-\gamma \log \gamma+\gamma \log \frac{d}{d-1}} .
\end{aligned}
$$

Consequently

$$
\begin{aligned}
\mathbf{E} H_{n} & =\sum_{k \geq 0} \mathbf{P}\left\{H_{n}>k\right\} \\
& \leq \tilde{\gamma} \log n+\sum_{k \geq \tilde{\gamma} \log n} \mathbf{P}\left\{H_{n}>k\right\} \\
& =\tilde{\gamma} \log n+O(1) .
\end{aligned}
$$

Remark. If $\Psi(w)$ is a polynomial of degree $d$ then the out-degrees of the corresponding increasing trees (with positive weight) are bounded by $d$. Hence we definitely have $H_{n} \gg \log n$ (and consequently $\mathbf{E} H_{n} \gg \log n$ ) which shows that the order of the upper bound for $\mathbf{E} H_{n}$ is best possible.

Lemma 5. Suppose that $y_{1}(z), y_{2}(z), \bar{y}_{1}(z), \bar{y}_{2}(z)$ are non-negative continuous functions that are defined for $z \geq 0$ such that $y_{1}(z)<\bar{y}_{1}(z)$ and $y_{2}(z)<\bar{y}_{2}(z)$ (at least) for a small interval $0<z<\zeta$ (for some $\zeta>0$ ). Further assume $y_{2}^{\prime}(z)=\Psi\left(y_{1}(z)\right)$, $\bar{y}_{2}^{\prime}(z)=\Psi\left(\bar{y}_{1}(z)\right)$ and that the difference $\bar{y}_{1}(z)-y_{1}(z)$ has exactly one positive zero. Then the difference $\bar{y}_{2}(z)-y_{2}(z)$ has at most one positive zero.

Proof. Suppose that $\bar{y}_{1}(z) \geq y_{1}(z)$ for $0 \leq z \leq z_{0}$ and $\bar{y}_{1}(z) \leq y_{1}(z)$ for $z \geq z_{0}$, that is, $z_{0}$ is the only (positive) zero of the difference $\bar{y}_{1}(z)-y_{1}(z)$. Since

$$
\begin{aligned}
\left(\bar{y}_{2}(z)-y_{2}(z)\right)^{\prime} & ==\Psi\left(\bar{y}_{1}(z)\right)-\Psi\left(y_{1}(z)\right) \\
& =\Psi^{\prime}(\xi(z))\left(\bar{y}_{1}(z)-y_{1}(z)\right)
\end{aligned}
$$

the same is true for the difference $\bar{y}_{2}^{\prime}(z)-y_{2}^{\prime}(z)$. Hence, the difference $\bar{y}_{2}(z)-y_{2}(z)$ is increasing for $0 \leq z \leq z_{0}$ and decreasing for $z \geq z_{0}$. Since $\bar{y}_{2}(0)>y_{2}(0)$ it directly follows that the difference $\bar{y}_{2}(z)-y_{2}(z)$ has at most one zero.

Remark . The assumption that $y_{1}(z), y_{2}(z), \bar{y}_{1}(z), \bar{y}_{2}(z)$ are non-negative can be weakened to the condition that $\Psi^{\prime}(w)>0$ for all $w \geq \min _{z \geq 0} \min \left\{y_{1}(z), \bar{y}_{1}(z)\right\}$.

Lemma 6. Suppose that $\mathbf{P}\left\{H_{n+1} \leq k\right\} \leq \mathbf{P}\left\{H_{n} \leq k\right\}$ for all $n$ and $k$. Then we have

and

$$
\mathbf{P}\left\{H_{n} \leq k\right\} \ll \frac{y_{k}(\rho)}{n^{\frac{1}{d-1}}}
$$

$$
\mathbf{P}\left\{H_{n}>k\right\} \ll \frac{n}{y_{k}(\rho)^{d-1}} .
$$


Proof. We define $\eta_{k}$ by $y\left(\rho\left(1-\eta_{k}\right)\right)=y_{k}(\rho)$. By Lemma 1 we have $\eta_{k} \sim c / y_{k}(\rho)^{d-1}$ (for some constant $c>0$ ).

Next observe that $y\left(z-\rho \eta_{k}\right)^{\prime}=\Psi\left(y\left(z-\rho \eta_{k}\right)\right)$ and that $\left.y\left(z-\rho \eta_{k}\right)\right|_{z=0}<y_{\ell}(0)$ for all $\ell$. Further, $y\left(z-\rho \eta_{k}\right)-y_{0}(z)=y\left(z-\rho \eta_{k}\right)$ has exactly one positive zero. Hence, by Lemma 5 it follows that $y\left(z-\rho \eta_{k}\right)-y_{\ell}(z)$ have at most one postive zero. (Note that $y\left(z-\rho \eta_{k}\right)$ is negative for $z<\rho \eta_{k}$. But for sufficiently large $k$ we surely have $\Psi^{\prime}\left(y\left(z-\rho \eta_{k}\right)\right)>0$ for all $z>0$, compare with the remark following Lemma 5.) In particular we know (by construction) that $y\left(\rho-\rho \eta_{k}\right)-y_{k}(\rho)=0$. Consequently $y\left(z-\rho \eta_{k}\right) \leq y_{k}(z)$ for $0 \leq z \leq \rho$ (and also $y\left(z-\rho \eta_{k}\right) \geq y_{k}(z)$ for $\left.\rho \leq z<\rho\left(1+\eta_{k}\right)\right)$.

First, set $z=\rho$. Since we have assumed that $y\left(z-\rho \eta_{k}\right) \leq y_{k}(z)$ for $0 \leq z \leq \rho$ we get

$$
\begin{aligned}
y_{k}(\rho) & =\sum_{\ell \geq 0} y_{\ell} \mathbf{P}\left\{H_{\ell} \leq k\right\} \frac{\rho^{\ell}}{\ell !} \\
& \geq \sum_{\ell \leq n} y_{\ell} \mathbf{P}\left\{H_{\ell} \leq k\right\} \frac{\rho^{\ell}}{\ell !} \\
& \geq \mathbf{P}\left\{H_{\ell} \leq k\right\} \sum_{\ell \leq n} y_{\ell} \frac{\rho^{\ell}}{\ell !} .
\end{aligned}
$$

Since $y_{\ell} \rho^{\ell} / \ell ! \sim c_{1} \ell^{\frac{1}{d-1}-1}$ (for some constant $c_{1}>0$ as $\ell \rightarrow \infty$ ) we immediately get $($ as $n \rightarrow \infty)$

$$
\sum_{\ell \leq n} y_{\ell} \frac{\rho^{\ell}}{\ell !} \sim c_{2} n^{\frac{1}{d-1}} .
$$

Thus, $\mathbf{P}\left\{H_{\ell} \leq k\right\} \ll y_{k}(\rho) n^{-\frac{1}{d-1}}$.

Next, let $x=\rho\left(1-\frac{1}{n}\right) \leq \rho$ and assume without loss of generality that $n \leq$ $y_{k}(\rho)^{d-1}$. Here we have

$$
\begin{aligned}
y(x)-y\left(x-\rho \eta_{k}\right) & \geq y(x)-y_{k}(x) \\
& \geq \sum_{\ell \geq n} y_{\ell} \mathbf{P}\left\{H_{\ell}>k\right\} \frac{x^{\ell}}{\ell !} \\
& \geq \mathbf{P}\left\{H_{n}>k\right\} \sum_{\ell \geq n} y_{\ell} \frac{x^{\ell}}{\ell !}
\end{aligned}
$$

It is now an easy exercise to show (with help of Lemma 1) that

$$
\sum_{\ell \geq n} y_{\ell} \frac{x^{\ell}}{\ell !} \gg \int_{n}^{\infty} y^{\frac{1}{d-1}-1} e^{-y / n} d y \gg n^{\frac{1}{d-1}}
$$

and that

$$
y(x)-y\left(x-\rho \eta_{k}\right) \sim c_{2} n^{\frac{1}{d-1}+1} \eta_{k}
$$

for some constant $c_{2}>0$. Of course, this implies $\mathbf{P}\left\{H_{n}>k\right\} \ll n / y_{k}(\rho)^{d-1}$ and completes the proof of the lemma.

By combining Lemma 5 and 6 we directly obtain Theorem 1 . Note that the assumption $y_{k+1}(\rho) \geq(1+\delta) y_{k}(\rho)$ combined with Lemma 6 provides exponential tail estimates of the kind

$$
\mathbf{P}\left\{\left|H_{n}-h_{n}\right| \geq \eta\right\} \ll e^{-\eta c_{4}},
$$

where $h_{n}=\max \left\{k: y_{k}(\rho)^{d-1} \leq n\right\}$ (and $c_{4}>0$ is a properly chosen constant). Of course, (9) implies

$$
\mathbf{E} H_{n}=h_{n}+O(1)
$$


and consequently

$$
\mathbf{P}\left\{\left|H_{n}-\mathbf{E} H_{n}\right| \geq \eta\right\} \ll e^{-\eta c_{4}}
$$

which also implies that $\operatorname{Var} H_{n}=O(1)$ as $n \rightarrow \infty$.

Note further that if the $\operatorname{limit}_{\lim _{k \rightarrow \infty}} y_{k+1}(\rho) / y_{k}(\rho)=\alpha$ exists then $h_{n}=$ $\max \left\{k: y_{k}(\rho)^{d-1} \leq n\right\} \sim(\log n) /(\log \alpha)$ and also

$$
\mathbf{E} H_{n} \sim \frac{\log n}{\log \alpha} .
$$

Note that we cannot prove $y_{k+1}(\rho) \geq(1+\delta) y_{k}(\rho)$ for all polynomial increasing tree families. However, the following lemma supports this conjectural relation and will also be used in Section 5 .

Lemma 7. There exists $\delta>0$ such that $y_{k}(\rho) \gg(1+\delta)^{k}$.

Proof. We choose $\gamma<1 / e$ and define $x_{k}$ by $y\left(x_{k}\right)=e^{\gamma k}$. Since $\Psi(w)$ is a polynomial of degree $d$ we, thus, get

$$
\Psi\left(y\left(x_{k}\right)\right) \leq C e^{\gamma k d}
$$

for some $C>0$. Furthermore

$$
\begin{aligned}
y\left(x_{k}\right)-y_{k}\left(x_{k}\right) & \leq \sum_{\ell \geq k} \frac{1}{\ell !}\left(\log \Psi\left(y\left(x_{k}\right)\right)\right)^{\ell} \\
& \leq \sum_{\ell \geq k} \frac{1}{\ell !}(\gamma k d+\log C)^{\ell} \\
& \ll \frac{(\gamma k d+\log C)^{k}}{k !} \\
& \ll \frac{(\gamma k d)^{k}}{k !}
\end{aligned}
$$

Hence, $y\left(x_{k}\right)-y_{k}\left(x_{k}\right) \rightarrow 0$ as $k \rightarrow \infty$. Conseqently with $\delta=\gamma-1$ we have

$$
y_{k}(\rho) \geq y_{k}\left(x_{k}\right)-y\left(x_{k}\right)+o(1) \gg e^{\gamma k}=(1+\delta)^{k} \text {. }
$$

Finally, we show that we definitely have $y_{k}(\rho) \ll(1+\bar{\delta})^{k}$ for some $\bar{\delta}>0$. Actually, there is a local version.

Lemma 8. There exists a constant $\bar{\delta}>0$ such that $y_{k+1}(\rho) \leq(1+\bar{\delta}) y_{k}(\rho)$ for all $k \geq 1$.

Proof. By monotonicity we have $y_{k}(x) \leq y(x)$ for $0 \leq x \leq \rho\left(1-\eta_{k}\right)$ and $y_{k}(x) \leq$ $y_{k}(c)$ for $\rho\left(1-\eta_{k}\right) \leq x \leq \rho$, where $\eta_{k}$ is defined by $y\left(\rho\left(1-\eta_{k}\right)\right)=y_{k}(\rho)$ and satisfies $\eta_{k} \sim c / y_{k}(\rho)^{d-1}$. From this we get

$$
\begin{aligned}
y_{k+1}(\rho) & \leq \int_{0}^{\rho\left(1-\eta_{k}\right)} \Psi(y(t)) d t+\rho \eta_{k} \Psi\left(y_{k}(\rho)\right) \\
& =y\left(\rho\left(1-\eta_{k}\right)\right)+\rho \eta_{k} \Psi\left(y_{k}(\rho)\right) \\
& \leq y_{k}(\rho)+\frac{C}{y_{k}(\rho)^{d-1}} y_{k}(\rho)^{d} \\
& \leq(1+C) y_{k}(\rho)
\end{aligned}
$$

for some constant $C>0$. 


\section{5. d-Ary Increasing Trees}

In this section we consider $d$-ary increasing trees defined by $\Psi(w)=(1+w)^{d}$. The special case $d=2$ has been discussed in [9]. Nevertheless it is not obvious to generalize this special case to the general one. In what follows we have tried to focus on those properties that are not easy to generalize whereas for the remaining parts we indicate the main lines and refer to the literature for technical details.

For $d$-ary incerasing trees we have $y(z)=(1-(d-1) z)^{-\frac{1}{d-1}}-1$ and $\rho=1 /(d-1)$. Further,

$$
y_{n}=n !(-(d-1))^{n}\left(\begin{array}{c}
-\frac{1}{d-1} \\
n
\end{array}\right) \sim n !(d-1)^{n} \frac{n^{\frac{2-d}{d-1}}}{\Gamma\left(\frac{1}{d-1}\right)} .
$$

As already mentioned in section 3, these kinds of trees surely satisfy

$$
\mathbf{P}\left\{H_{n+1} \leq k\right\} \leq \mathbf{P}\left\{H_{n} \leq k\right\} .
$$

Thus, in order to apply Theorem 1 (unconditionally) we have to show that there exists $\delta>0$ with $y_{k+1}(\rho) \geq(1+\delta) y_{k}(\rho)$. In fact, we will prove much more.

For convenience, we set $\tilde{y}(z)=y(z)+1$ and $\tilde{y}_{k}(z)=y_{k}(z)+1$. These functions satisfy $\tilde{y}^{\prime}(z)=\tilde{y}(z)^{d}$ and $\tilde{y}_{k+1}^{\prime}(z)=\tilde{y}_{k}(z)^{d}$ with initial values $\tilde{y}_{k}(0)=\tilde{y}(0)=1$ and $\tilde{y}_{0}(z) \equiv 1$.

Lemma 9. For all $k \geq 0$ we have

$$
\frac{\tilde{y}_{k+2}(\rho)}{\tilde{y}_{k+1}(\rho)} \leq \frac{\tilde{y}_{k+1}(\rho)}{\tilde{y}_{k}(\rho)} .
$$

Proof. For $0 \leq \gamma<1$ set

$$
v_{k}(z, \gamma)= \begin{cases}\tilde{y}(z) & \text { for } 0 \leq z \leq \rho(1-\gamma) \\ \gamma^{-1 /(d-1)} \tilde{y}_{k}\left(\frac{z-\rho(1-\gamma)}{\gamma}\right) & \text { for } \rho(1-\gamma) \leq z \leq \rho\end{cases}
$$

These functions satisfy

$$
v_{k+1}(z, \gamma)=v_{k}(z, \gamma)^{d}
$$

$v_{k}(0)=1$ and $v_{k}(\rho, \gamma)=\gamma^{-1 /(d-1)} \tilde{y}_{k}(\rho)$. In particular, for $\gamma_{k}=\left(\tilde{y}_{k+1}(\rho) / \tilde{y}_{k}(\rho)\right)^{d-1}$ we have

$$
v_{k}\left(\rho, \gamma_{k}\right)=\tilde{y}_{k+1}(\rho) \text {. }
$$

Now inductive application of Lemma 5 shows that $\tilde{y}_{k+1}(z)-v_{k}(z, \rho)$ have (at most) one positive zero. In particular we get

$$
\tilde{y}_{k+1}(z) \leq v_{k}\left(z, \gamma_{k}\right) \quad \text { for } \quad 0 \leq z \leq \rho .
$$

By integration this also implies that

$$
\tilde{y}_{k+2}(z) \leq v_{k+1}\left(z, \gamma_{k}\right) \text { for } 0 \leq z \leq \rho
$$

and consequently for $z=\rho$ :

$$
\tilde{y}_{k+2}(\rho) \leq v_{k+1}\left(\rho, \gamma_{k}\right)=\gamma_{k}^{-1 /(d-1)} \tilde{y}_{k+1}(\rho)=\frac{\tilde{y}_{k+1}(\rho)^{2}}{\tilde{y}_{k}(\rho)}
$$

which completes the proof of Lemma 9

Corollary . There exists $\alpha>1$ with

$$
\alpha=\lim _{k \rightarrow \infty} \frac{y_{k+1}(\rho)}{y_{k}(\rho)} .
$$

Proof. Lemma 9 implies that there exists $\alpha \geq 1$ with

$$
\alpha=\lim _{k \rightarrow \infty} \frac{\tilde{y}_{k+1}(\rho)}{\tilde{y}_{k}(\rho)} .
$$

Since $y_{k}(\rho) \rightarrow \infty$ this also implies (10). Finally, Lemma 7 implies that $\alpha>1$. 
This already proves $\mathbf{E} H_{n} \sim c \log n$ (for some constant $c=1 /(\log \bar{\alpha})>0$ ) and the concentration property $\mathbf{P}\left\{\left|H_{n}-\mathbf{E} H_{n}\right| \geq \eta\right\} \ll e^{-c \eta}$.

Our next aims are to show that $\bar{\alpha}=e^{1 / c_{d}}$, where $c_{d}$ is defined in (1) and to prove the limit relation (2). For both purposes we will use the solution of the integral equation (3). Then we will introduce properly defined auxiliary functions $\bar{y}_{k}(\alpha, z)$. The procedure is almost the same as that of [9]. Therefore we will not work out all details.

Note that it was already shown by Broutin et al. [2] that $H_{n} /(\log n) \rightarrow c_{d}$ in probability. This result is, of course, in accordance with $\mathbf{E} H_{n} \sim c_{d} \log n$.

Lemma 10. Set $\alpha_{0}=e^{1 / c_{d}}$, where $c_{d}$ is defined in (1). Suppose that $1<\alpha<\alpha_{0}$ and that $\beta<\gamma:=c_{d}-1 /(d-1)$ denotes the smallest positive solution of

$$
\alpha^{\beta}=\frac{\beta(d-1)+1}{d} .
$$

Further, let $\mathcal{F}$ denote the set of monotonely decreasing and continuous functions $F(y)(y \geq 0)$ that satisfy

$$
F(y)=1-y^{\beta}+O\left(y^{\gamma}\right) \quad(y \rightarrow 0+)
$$

and $F(y) \rightarrow 0$ as $y \rightarrow \infty$. Then there exists a unique solution $F \in \mathcal{F}$ of the integral equation

$$
y^{\frac{1}{d-1}} F(y / \alpha)=\frac{\Gamma\left(\frac{d}{d-1}\right)}{\Gamma\left(\frac{1}{d-1}\right)^{d}} \int_{y_{1}+\cdots+y_{d}=y, y_{j} \geq 0} \prod_{j=1}^{d}\left(F\left(y_{j}\right) y_{j}^{\frac{1}{d-1}-1}\right) d \mathbf{y} .
$$

Moreover, there exist $C>0$ and $\kappa>0$ such that

$$
F(y)=O\left(e^{-C y^{\kappa}}\right)
$$

as $y \rightarrow \infty$

Proof. It is easy to show that $\rho$ defined by

$$
\rho\left(F_{1}, F_{2}\right)=\sup _{y \geq 0}\left(\left|F_{1}(y)-F_{2}(y)\right| y^{-\gamma}\right)
$$

is a complete metric on $\mathcal{F}$ (compare with [9]) and that (12) is a contracting fixed point equation on $\mathcal{F}$ that can be rewritten as $F=A(F)$ (with Lipschitz constant $\left.L=d \alpha^{\gamma} /(1+\gamma(d-1))<1\right)$. Hence, by Banach's fixed point theorem there is a unique solution.

Finally, (13) can be proved in an inductive way. Let $F_{0}(y)=\max \left\{1-y^{\beta}, 0\right\}$ and $F_{n+1}=A\left(F_{n}\right)$. Then $F_{n} \rightarrow F$ and (by the same method as in [9]) one can show (inductively) that $F_{n}(y) \leq C e^{-y^{\kappa}}$ (for $y \geq 1$ and some constant $C>0$ ), where $\kappa=(\log d)(\log d-\log \alpha)>0$.

Note that for every scaling factor $c>0$ the function $F(c y)$ is also a solution of (12). Thus, we can assume (without loss of generality) that there is $F_{\alpha}$ that satisfies (12) and

$$
\int_{0}^{\infty} F_{\alpha}(y) y^{\frac{1}{d-1}-1} d y=(d-1)^{\frac{1}{d-1}} \Gamma\left(\frac{1}{d-1}\right) .
$$

Note further that $\alpha=\alpha_{0}$ is critical value for the fixed point equation (12) because the Lipschitz constant $L$ would equal 1 and, thus, we cannot apply Banach's fixed point theorem. Nevertheless it will possible to solve (12) even for $\alpha=\alpha_{0}$, see Lemma 14.

We introduce the Laplace transforms

$$
\Phi_{\alpha}(u)=\frac{1}{(d-1)^{\frac{1}{d-1}} \Gamma\left(\frac{1}{d-1}\right)} \int_{0}^{\infty} F_{\alpha}(y) y^{\frac{1}{d-1}-1} e^{-u y} d y .
$$


These are entire functions and are also given by $\Phi_{\alpha}(0)=1$ and by the differential equation

$$
\Phi_{\alpha}^{\prime}(u)=-\frac{1}{\alpha^{d /(d-1)}} \Phi_{\alpha}\left(\frac{u}{\alpha}\right)^{d} .
$$

Note that if $\alpha>1$ then (15) has always a unique (entire) solution. It is easy to find a (formal) power series expansion $\Phi_{\alpha}(u)=\sum_{k} c_{k} u^{k}$ which is also convergent in the whole complex plane (see [8]). Furthermore, we definitely have $\lim _{\alpha \rightarrow \alpha_{0}} \Phi_{\alpha}(u)=$ $\Phi_{\alpha_{0}}(u)$ (uniformly for $u$ in a compact subset of the complex plane).

We define auxiliary functions

$$
\bar{y}_{k}(\alpha, z)=\alpha^{k /(d-1)} \Phi_{\alpha}\left(\alpha^{k}(\rho-z)\right),
$$

where $k$ can be considered a real (not necessarily integral) parameter. The next lemma collects some useful facts on $\bar{y}_{k}(z)$. We omit the proof. All properties are direct consequences of the definition and the properties of $F_{\alpha}$ (see also [9]).

Lemma 11. Suppose that $1<\alpha<\alpha_{0}=e^{1 / c_{d}}$ and $\beta$ be given by (11). The functions $\bar{y}_{k}(\alpha, z)$ be defined by (16). have the following properties.

(1) For all $k>0$ the function $\bar{y}_{k}(\alpha, z)$ in monotone for $z \geq 0$. The initial value satisfies $0<\bar{y}_{k}(\alpha, 0)<1$, more precisely we have $1-\bar{y}_{k}(\alpha, 0) \sim C \alpha^{-\beta k}$ for some constant $C$ depending on $\alpha$. Furthermore $\bar{y}_{k}(\alpha, \rho)=\alpha^{k /(d-1)}$.

(2) The functions $\bar{y}_{k}(\alpha, z)$ satisfy the recurrence relation

$$
\bar{y}_{k+1}^{\prime}(\alpha, z)=\bar{y}_{k}(\alpha, z)^{d} .
$$

(3) For all integers $\ell \geq 0$ and for all real numbers $k>0$ the difference $\tilde{y}_{\ell}(z)-$ $\bar{y}_{k}(\alpha, z)$ has exactly one positive zero $z_{\ell, k}$. In particular we have $\bar{y}_{k}(\alpha, z) \leq$ $\tilde{y}_{\ell}(z)$ for $0 \leq z \leq z_{\ell, k}$ and $\bar{y}_{k}(\alpha, z) \geq \tilde{y}_{\ell}(z)$ for $z \geq z_{\ell, k}$.

As a first application we provide an asymptotic expansion for the expected height E $H_{n}$.

Lemma 12. We have $\lim _{k \rightarrow \infty} y_{k+1}(\rho) / y_{k}(\rho)=e^{1 /\left(c_{d}(d-1)\right)}$ and consequently

$$
\mathbf{E} H_{n} \sim c_{d} \log n \text {. }
$$

Proof. Suppose that $1<\alpha<\alpha_{0}$ and set $e_{k}:=(d-1)\left(\log \tilde{y}_{k}(\rho)\right) /(\log \alpha)$. Then the function $\bar{y}_{e_{k}}(\alpha, z)$ satisfies $\bar{y}_{e_{k}}(\alpha, 0)<y_{k}(0)$ and

$$
\bar{y}_{e_{k}}(\alpha, \rho)=\tilde{y}_{k}(\rho) \text {. }
$$

Hence, by Lemma 11 it follows that $\bar{y}_{e_{k}}(\alpha, z) \leq \tilde{y}_{k}(z)$ for $0 \leq z \leq \rho$. Consequently by integration it also follows that $\bar{y}_{e_{k}+1}(\alpha, z) \leq \tilde{y}_{k+1}(z)$ for $0 \leq z \leq \rho$. In particular,

$$
\bar{y}_{e_{k}+1}(\alpha, \rho)=\alpha^{\frac{1}{d-1}} \tilde{y}_{k}(\rho) \leq \tilde{y}_{k+1}(\rho)
$$

Thus, we have $\tilde{y}_{k+1}(\rho) / \tilde{y}_{k}(\rho) \geq \alpha^{1 /(d-1)}$ for all $\alpha<\alpha_{0}$ and consequently $\tilde{y}_{k+1}(\rho) / \tilde{y}_{k}(\rho) \geq \alpha_{0}^{1 /(d-1)}=e^{1 / c_{d}}$

In a second (and much more involved) step we will show that for every $\varepsilon>0$

$$
\log \tilde{y}_{k}(\rho) \leq \frac{k}{c_{d}(d-1)}(1+\varepsilon)
$$

for sufficiently $k \geq k_{0}(\varepsilon)$. Since we already know that the limit $\lim _{k \rightarrow \infty} y_{k+1}(\rho) / y_{k}(\rho)=\bar{\alpha}$ exists it follows that $\bar{\alpha}=e^{1 /\left(c_{d}(d-1)\right)}$. Finally, since $\mathbf{E} H_{n}=\max \left\{k: \tilde{y}_{k}(\rho)^{d-1} \leq n\right\}+O(1)$ this also give (17).

We again fix $\alpha<\alpha_{0}$ and define $t(\alpha)>0$ by

$$
(1+t(\alpha)) \alpha^{\beta} \log \alpha=\frac{d-1}{d} .
$$

Note that $\lim _{\alpha \rightarrow \alpha_{0}} t(\alpha)=0$. 
Further, set $\delta_{k}(z)=\tilde{y}_{k}(z)-\bar{y}_{k+r}(\alpha, z)$, where $r \geq 0$ is a parameter that will be chosen appropriately. Note that $\tilde{y}_{k}(z) \leq \tilde{y}(z)$ and $\bar{y}_{k+r}(\alpha, z) \leq \tilde{y}(z)$ for $0 \leq z<\rho=$ $1 /(d-1)$. By induction it follows that

$$
\delta_{k}(z) \leq \sum_{\ell=0}^{k} \delta_{\ell}(0) \frac{\left(\frac{d}{d-1} L(z)\right)^{k-\ell}}{(k-\ell) !}
$$

where $L=\log 1 /(1-(d-1) z)$, compare with the proof of Lemma 2. We now suppose that $r=2 k t(\alpha)$, set $z^{\prime}=\frac{1}{d-1}-\alpha^{-k(1+t(\alpha))}$ and estimate $\tilde{y}_{k}\left(z^{\prime}\right)=\bar{y}_{k+2 k t(\alpha)}\left(\alpha, z^{\prime}\right)+$ $\delta_{k}\left(z^{\prime}\right)$ from above. We have

$$
\begin{aligned}
\bar{y}_{k+2 k t(\alpha)}\left(\alpha, z^{\prime}\right) & =\alpha^{\frac{k(1+t(\alpha))}{d-1}} \alpha^{\frac{k t(\alpha)}{d-1}} \Phi_{\alpha}\left(\alpha^{k t(\alpha)} \rho\right) \\
& =\alpha^{\frac{k(1+t(\alpha))}{d-1}}\left(1-\frac{C}{\alpha^{\beta k t(\alpha)}}(1+o(1))\right)
\end{aligned}
$$

and

$$
\begin{aligned}
\delta_{k}\left(z^{\prime}\right) & \leq \sum_{\ell=0}^{k} \frac{C}{\alpha^{\beta \ell+2 \beta k t(\alpha)}} \frac{\left(\frac{d}{d-1} k(1+t(\alpha)) \log \alpha\right)^{k-\ell}}{(k-\ell) !} \\
& =\frac{C}{\alpha^{\beta k(1+2 t(\alpha))}} \sum_{\ell=0}^{k} \frac{\left(\frac{d}{d-1} k(1+t(\alpha)) \alpha^{\beta} \log \alpha\right)^{k-\ell}}{(k-\ell) !} \\
& =\frac{C}{\alpha^{\beta k(1+2 t(\alpha))}} \sum_{\ell=0}^{k} \frac{k^{k-\ell}}{(k-\ell) !} \\
& \sim \frac{C}{\alpha^{\beta k(1+2 t(\alpha))}} \frac{e^{k}}{2} \\
& =\frac{1}{2} \alpha^{\frac{k(1+t(\alpha))}{d-1}} \frac{C}{\alpha^{\beta k t(\alpha)}}
\end{aligned}
$$

and consequently

$$
\tilde{y}_{k}\left(z^{\prime}\right) \leq \alpha^{\frac{k(1+t(\alpha))}{d-1}}\left(1-\frac{1}{2} \frac{C}{\alpha^{\beta k t(\alpha)}}(1+o(1))\right) .
$$

If we compare that with

$$
\bar{y}_{k+3 k t(\alpha)}\left(\alpha, z^{\prime}\right)=\alpha^{\frac{k(1+t(\alpha))}{d-1}}\left(1-\frac{C}{\alpha^{2 \beta k t(\alpha)}}(1+o(1))\right)
$$

we observe that (for sufficiently large $k$ )

$$
\tilde{y}_{k}\left(z^{\prime}\right) \leq \bar{y}_{k+3 k t(\alpha)}\left(\alpha, z^{\prime}\right) \text {. }
$$

Since $1=\tilde{y}_{k}(0)>\bar{y}_{k+3 k t(\alpha)}(\alpha, 0)$ it follows from Lemma 11 that $\tilde{y}_{k}(z) \leq$ $\bar{y}_{k+3 k t(\alpha)}(\alpha, z)$ even for all $z \geq z^{\prime}$. In particular we have (for sufficiently large $k$ )

$$
\tilde{y}_{k}(\rho) \leq \bar{y}_{k+3 k t(\alpha)}(\alpha, \rho)=\alpha^{k(1+3 t(\alpha)) /(d-1)} \leq e^{k(1+3 t(\alpha)) / c_{d}} .
$$

Since we can choose $\alpha$ that $t(\alpha)$ is arbitrarily small this completes the proof of the lemma.

Remark. With a litte bit more care we can be precise. Following the ideas of [7], that is, taking into account the dependence of $F_{\alpha}$ on $\alpha$ as $\alpha \rightarrow \alpha_{0}$ one gets $k /\left(c_{d}(d-1)\right) \leq \log y_{k}(\rho) \leq k /\left(c_{d}(d-1)\right)+O(\sqrt{k} \cdot \log k)$. Of course, this translates to

$$
\mathbf{E} H_{n}=c_{d} \log n+O(\sqrt{\log n}(\log \log n)) .
$$

However, this bound is surely not best possible. As in the binary case one expects that $\mathbf{E} H_{n}=c_{d} \log n-c_{d}^{\prime} \log \log n+O(1)$. Since we are far away from optimality 
we have decided not to include a full proof of (18) but have restricted ourselves to (17).

Lemma 13. Set $\alpha_{0}=e^{1 / c_{d}}$. Then we have

$$
\lim _{k \rightarrow \infty} \frac{1}{\tilde{y}_{k}(\rho)} \tilde{y}_{k}\left(\rho-\frac{u}{\tilde{y}_{k}(\rho)^{d-1}}\right)=\Phi_{\alpha_{0}}(u) .
$$

Furthermore, for every $1<\alpha<\alpha_{0}$

$$
\Phi_{\alpha}(u) \leq \Phi_{\alpha_{0}}(u) \quad \text { for } u>0
$$

and

$$
\Phi_{\alpha}(u) \geq \Phi_{\alpha_{0}}(u) \quad \text { for } u<0
$$

Proof. Set

$$
V_{\ell, k}(u)=\frac{1}{\tilde{y}_{k}(\rho)} \tilde{y}_{\ell}\left(\rho-\frac{u}{\tilde{y}_{k}(\rho)^{d-1}}\right) .
$$

By definition these functions satisfy $V_{\ell+1, k}^{\prime}(u)=-V_{\ell, k}(u)^{d}$.

Our first aim is to show that $V_{\ell+1, k+1}(u)-V_{\ell, k}(u)$ has (at most) one zero for $u \leq \tilde{y}_{k}(\rho)^{d-1}$. This is obviously true for $\ell=0$ ( since $\tilde{y}_{0}(u)=1$ and $\left.\tilde{y}_{1}(u)=1+u\right)$. Next observe that

$$
V_{\ell, k}\left(-\tilde{y}_{k}(\rho)^{d-1}\right)=\frac{1}{\tilde{y}_{k}(\rho)}
$$

and (by using $\tilde{y}_{\ell+1}(u) \leq \tilde{y}(u)$ for $u \geq 0$ )

$V_{\ell+1, k+1}\left(-\tilde{y}_{k}(\rho)^{d-1}\right)=\frac{1}{\tilde{y}_{k+1}(\rho)} \tilde{y}_{\ell+1}\left(\rho-\frac{\tilde{y}_{k}(\rho)^{d-1}}{\tilde{y}_{k+1}(\rho)^{d-1}}\right) \leq \frac{1}{\tilde{y}_{k+1}(\rho)} \frac{\tilde{y}_{k+1}(\rho)}{\tilde{y}_{k}(\rho)}=\frac{1}{\tilde{y}_{k}(\rho)}$ we can proceed as in the proof of Lemma 5 .

Since $V_{k, k}(0)=V_{k+1, k+1}(0)$ we get

$$
V_{k, k}(u)<V_{k+1, k+1}(u) \text { for } u<0
$$

and

$$
V_{k, k}(u)>V_{k+1, k+1}(u) \text { for } u>0 .
$$

Hence, there exists a function $V(u)$ with $V(u)=\lim _{k \rightarrow \infty} V_{k, k}(u)$. Note that

$$
V_{k+1, k+1}^{\prime}(u)=-\frac{1}{\alpha_{k}^{d /(d-1)}} V_{k, k}\left(\frac{u}{\alpha_{k}}\right)^{d},
$$

where $\alpha_{k}=\left(\tilde{y}_{k+1}(\rho) / \tilde{y}_{k}(\rho)\right)^{d-1}$ converges (monotonely) to $\alpha_{0}=e^{1 / c_{d}}$. Since $V(u)$ is monotone this also shows that $\sup _{k \geq 1} \sup _{u_{1} \leq u \leq u_{2}} V_{k, k}^{\prime}(u)$ is bounded for each interval $\left[u_{1}, u_{2}\right]$. Consequently $V(u)$ is continuous and, thus, (19) implies that

$$
V^{\prime}(u)=-\frac{1}{\alpha_{0}^{d /(d-1)}} V\left(\frac{u}{\alpha_{0}}\right)^{d} .
$$

Since $V(0)=1$ this also shows that $V(u)=\Phi_{\alpha_{0}}(u)$.

Now fix some $1<\alpha<\alpha_{0}$ and define $e_{k}=(d-1)\left(\log \tilde{y}_{k}(\rho)\right) /(\log \alpha)$. By Lemma 11 the function

satisfies

$$
\Phi_{\alpha}(u)=\frac{1}{\alpha^{\frac{e_{k}}{d-1}}} \bar{y}_{e_{k}}\left(\rho-\frac{u}{\alpha^{e_{k}}}\right)
$$

$$
V_{k, k}(u)<\Phi_{\alpha}(u) \quad \text { for } u<0
$$

and

$$
V_{k, k}(u)>\Phi_{\alpha}(u) \text { for } u>0 .
$$

Hence, the same inequalities are satisfied for the limit $V(u)=\Phi_{\alpha_{0}}(u)$, too. 
Lemma 14. Let $\alpha=\alpha_{0}=e^{1 / c_{d}}$. Then (12) has a unique continuous and monotonely decreasing solution $F_{\alpha_{0}}$ that satisfies

$$
\Phi_{\alpha_{0}}(u)=\frac{1}{(d-1)^{\frac{1}{d-1}} \Gamma\left(\frac{1}{d-1}\right)} \int_{0}^{\infty} F_{\alpha_{0}}(y) y^{\frac{1}{d-1}-1} e^{-u y} d y .
$$

Furthermore, we have

$$
1-F_{\alpha_{0}}(y)=O\left(y^{\beta}\right) \quad(y \rightarrow 0+)
$$

for all $\beta<c_{d}-1 /(d-1)$ and

$$
F_{\alpha_{0}}(y)=O\left(e^{-C y^{\kappa}}\right) \quad(y \rightarrow \infty)
$$

for some $C>0$ and $\kappa>0$.

Proof. We want to define $F_{\alpha_{0}}(y)=\lim _{\alpha \rightarrow \alpha_{0}} F_{\alpha}(y)$. Since

$$
F_{\alpha}(y)=y^{1-\frac{1}{d-1}} \frac{(d-1)^{\frac{1}{d-1}} \Gamma\left(\frac{1}{d-1}\right)}{2 \pi i} \int_{-\infty}^{\infty} \Phi_{\alpha}(i t) e^{i y t} d t
$$

we can do this formally by interchanging the limit and the integral. In order to justify this formal procedure we proceed similarly to [9]. Since the integral is not absolutely convergent we have to use partial integration to replace it by the inverse Laplace transform of $\Phi^{\prime}(u)$ etc. (we omit the technical details). In particular we get (20).

Next set $\Phi_{\infty}(u):=\Gamma\left(\frac{1}{d-1}\right) u^{-\frac{1}{d-1}}$ and suppose that $u>0$. Then we get from Lemma 13

$$
\begin{aligned}
\Phi_{\infty}(u)-\Phi_{\alpha}(u) & \geq \Phi_{\infty}(u)-\Phi_{\alpha_{0}}(u) \\
& =\frac{1}{(d-1)^{\frac{1}{d-1}} \Gamma\left(\frac{1}{d-1}\right)} \int_{0}^{\infty}\left(1-F_{\alpha_{0}}(y)\right) y^{\frac{1}{d-1}-1} e^{-u y} d y \\
& \geq \frac{1-F_{\alpha_{0}}(u)}{(d-1)^{\frac{1}{d-1}} \Gamma\left(\frac{1}{d-1}\right)} \int_{u}^{\infty} y^{\frac{1}{d-1}-1} e^{-u y} d y \\
& \gg\left(1-F_{\alpha_{0}}(u)\right) u^{-\frac{1}{d-1}} .
\end{aligned}
$$

Since $\Phi_{\infty}(u)-\Phi_{\alpha}(u) \sim C u^{-\frac{1}{d-1}-\beta}$ we directly obtain (21).

In a similar way we get (for $u<0$ and any $y_{0}>0$ )

$$
\begin{aligned}
\Phi_{\alpha}(u) & \geq \Phi_{\alpha_{0}}(u) \\
& =\frac{1}{(d-1)^{\frac{1}{d-1}} \Gamma\left(\frac{1}{d-1}\right)} \int_{0}^{\infty} F_{\alpha_{0}}(y) y^{\frac{1}{d-1}-1} e^{-u y} d y \\
& \geq \frac{F_{\alpha_{0}}\left(y_{0}\right)}{(d-1)^{\frac{1}{d-1}} \Gamma\left(\frac{1}{d-1}\right)} \int_{0}^{y_{0}} y^{\frac{1}{d-1}-1} e^{-u y} d y .
\end{aligned}
$$

Since $\Phi(u) \ll e^{(-u)^{\delta}}$ (for some $\delta>1$ ) we get the upper bound (22) for all $\kappa<$ $1 /(\delta-1)$.

With help of $\Phi_{\alpha_{0}}(u)$ we can also work with the auxiliary functions $\bar{y}_{k}\left(\alpha_{0}, z\right)$.

Lemma 15. The functions $\bar{y}_{k}\left(\alpha_{0}, z\right)$ are entire functions. Furthermore the Taylor coefficients of $\bar{y}_{k}\left(\alpha_{0}, z\right)=\sum_{n \geq 0} \bar{y}_{k, n}\left(\alpha_{0}\right) z^{n} / n$ ! are given by

$$
\bar{y}_{k, n}\left(\alpha_{0}\right)=\frac{(d-1)^{n}}{\Gamma\left(\frac{1}{d-1}\right)} \int_{0}^{\infty} F_{\alpha_{0}}\left((d-1) v \alpha_{0}^{-k}\right) v^{\frac{1}{d-1}-1+n} e^{-v} d v
$$


and asymptotically by

$$
\bar{y}_{k, n}\left(\alpha_{0}\right)=y_{n} F_{\alpha_{0}}\left((d-1) n \alpha_{0}^{-k}\right)+o\left(y_{n}\right),
$$

where the error term is uniform for $n \geq 0$ as $k \rightarrow \infty$.

Proof. Since $\Phi_{\alpha_{0}}(u)$ is entire, the same is true for $\bar{y}_{k}\left(\alpha_{0}, z\right)$. Next, the explicit expression for $\bar{y}_{k, n}\left(\alpha_{0}\right)$ follows immediately from the definition of $\bar{y}_{k}\left(\alpha_{0}, z\right)$ and from $(20)$.

Finally, the kernel $v^{n} e^{-v}$ is concentrated in the interval $\left[n-n^{\frac{1}{2}+\varepsilon}, n+n^{\frac{1}{2}+\varepsilon}\right]$ and consequently the factor $F_{\alpha_{0}}\left((d-1) v \alpha_{0}^{-k}\right) v^{\frac{1}{d-1}-1}$ can be asymptotically replaced by $F_{\alpha_{0}}\left((d-1) n \alpha_{0}^{-k}\right) n^{\frac{1}{d-1}-1}$. If $c_{1} \leq n / \alpha_{k} \leq c_{2}$ (for some arbitrary constants) then (23) is surely uniform. By combining (23) for $c_{1} \leq n / \alpha_{k} \leq c_{2}$ with the tail estimates of $F$ (from Lemma 14) we can extend this uniformity to all $n \geq 0$ as $k \rightarrow \infty$.

Finally, we provide an approximation for $\mathbf{P}\left\{H_{n} \leq k\right\}$. The idea is to show that $\tilde{y}_{k}(z)$ can be properly approximated by $\bar{y}_{e_{k}}\left(\alpha_{0}, z\right)$ in a complex neighbourhood of $z=\rho$, where $e_{k}=(d-1)\left(\log \tilde{y}_{k}(\rho)\right) /\left(\log \alpha_{0}\right)$. (Note that by definition $\tilde{y}_{k}(\rho)=$ $\bar{y}_{e_{k}}\left(\alpha_{0}, \rho\right)$.) It then follows by Cauchy's formula that also the Taylor coefficients are comparable. (We do not work out details since exactly the same procedure has been applied in [9] for the special case $d=2$.) Hence, $y_{n} \mathbf{P}\left\{H_{n} \leq k\right\}$ is approximated by $\bar{y}_{e_{k}, n}\left(\alpha_{0}\right)$. Note that Lemma 15 says that $\bar{y}_{e_{k}, n}\left(\alpha_{0}\right)$ is approxmated by

$$
y_{n} F_{\alpha_{0}}\left((d-1) n \alpha_{0}^{-e_{k}}\right)=y_{n} F_{\alpha_{0}}\left((d-1) n / \tilde{y}_{k}(\rho)^{d-1}\right) .
$$

This completes the proof of Theorem 2 .

\section{Recursive Trees}

Recursive trees are defined by $\Psi(w)=e^{w}$. Thus, they do not constitute a polynomial increasing tree family. Nevertheless they behave very similarly to $d$-ary increasing trees. (However, the generating functioin $y(z)=\log (1 /(1-z))=\sum_{n \geq 1} z^{n} / n$ has a logarithmic singularity and not an algebraic one.)

The distribution of the height is encoded by the generating functions $y_{k}(z)=$ $\sum_{n \geq 0} \mathbf{P}\left\{H_{n} \leq k\right\} z^{n} / n$ that are given by $y_{0}(z)=0$ and recusively by

$$
y_{k+1}^{\prime}(z)=e^{y_{k}(z)} \quad\left(y_{k+1}(0)=0\right) .
$$

In what follows It will be (partly) more convenient to work instead with

$$
Y_{k}(z)=y_{k}^{\prime}(z)=\sum_{n \geq 0} \mathbf{P}\left\{H_{n+1} \leq k\right\} z^{n} .
$$

Here we have $Y_{1}(z)=1$ and

$$
Y_{k+1}^{\prime}(z)=Y_{k+1}(z) Y_{k}(z) \quad\left(Y_{k+1}(0)=1\right) .
$$

Thus, $Y_{k}(z)$ looks similar to $\bar{y}_{k}(z)$ in the case of binary increasing trees. Therefore it is not unexpected that the asymptotic behaviour is (almost) the same as for $d$ ary increasing trees. In fact, one can proceed (almost) in the same way as above. However, there are several (non-trivial) differences. In what follows we will focus on these differences and will not work out all details.

We start with an analogue to Lemma 5.

Lemma 16. Suppose that $Y_{1}(z), Y_{2}(z), \bar{Y}_{1}(z), \bar{Y}_{2}(z)$ are non-negative continuous functions that are defined for $z \geq 0$ such that $Y_{1}(0)<\bar{Y}_{1}(0), Y_{2}(0)<\bar{Y}_{2}(0)$, $Y_{2}^{\prime}(z)=Y_{2}(z) Y_{1}(z), \bar{Y}_{2}^{\prime}(z)=\bar{Y}_{2}(z) \bar{Y}_{1}(z)$, and that the difference $\bar{Y}_{1}(z)-Y_{1}(z)$ has exactly one positive zero. Then the difference $\bar{Y}_{2}(z)-Y_{2}(z)$ has at most one positive zero. 
Proof. For $j=1,2$ set

$$
y_{j}(z)=\int_{0}^{z} Y_{j}(t) d t \quad \text { and } \quad \bar{y}_{j}(z)=\int_{0}^{z} \bar{Y}_{j}(t) d t .
$$

Then we have $y_{1}(z)<\bar{y}_{1}(z), y_{2}(z)<\bar{y}_{2}(z)$ (at least) for a small interval $0<z<\zeta$ and also $y_{2}^{\prime}(z)=e^{y_{1}(z)}$ and $\bar{y}_{2}^{\prime}(z)=e^{\bar{y}_{1}(z)}$. Furthermore, since $\bar{Y}_{1}(z)-Y_{1}(z)$ is positive (for small positive $z$ ) and has at most one positive zero, the same follows for

$$
\bar{y}_{1}(z)-y_{1}(z)=\int_{0}^{z}\left(\bar{Y}_{1}(t)-Y_{1}(t)\right) d t
$$

compare with the proof of Lemma 5 . Now observe that $\left(e^{y}-e^{z}\right) /(y-z)>0$ for real $y, z$ with $y \neq z$. Hence,

$$
\bar{Y}_{2}(z)-Y_{2}(z)=\bar{y}_{2}^{\prime}(z)-y_{2}^{\prime}(z)=e^{\bar{y}_{1}(z)}-e^{y_{1}(z)}=\frac{e^{\bar{y}_{1}(z)}-e^{y_{1}(z)}}{\bar{y}_{1}(z)-y_{1}(z)}\left(\bar{y}_{1}(z)-y_{1}(z)\right)
$$

has at most one positive zero, too.

The next property is an analogue to Lemma 9.

Lemma 17. For all $k \geq 0$ we have

$$
\frac{Y_{k+2}(1)}{Y_{k+1}(1)} \leq \frac{Y_{k+1}(1)}{Y_{k}(1)}
$$

Proof. We proceed as in the proof of Lemma 9. For $0 \leq \gamma<1$ set

$$
V_{k}(z, \gamma)= \begin{cases}\frac{1}{1-z} & \text { for } 0 \leq z \leq 1-\gamma, \\ \gamma^{-1} Y_{k}\left(\frac{z-(1-\gamma)}{\gamma}\right) & \text { for } 1-\gamma \leq z \leq \rho .\end{cases}
$$

These functions satisfy

$$
V_{k+1}(z, \gamma)=V_{k+1}(z, \gamma) V_{k}(z, \gamma),
$$

$V_{k}(0)=1$ and $V_{k}(1, \gamma)=\gamma^{-1} Y_{k}(1)$. In particular, for $\gamma_{k}=Y_{k+1}(1) / Y_{k}(1)$ we have $V_{k}\left(1, \gamma_{k}\right)=Y_{k+1}(1)$. Now inductive application of Lemma 16 shows that $Y_{k+1}(z)-$ $V_{k}(z, \rho)$ have (at most) one positive zero. In particular we get

$$
Y_{k+1}(z) \leq V_{k}\left(z, \gamma_{k}\right) \text { for } \quad 0 \leq z \leq 1
$$

and after integration

$$
Y_{k+2}(1) \leq V_{k+1}\left(1, \gamma_{k}\right)=\gamma_{k}^{-1} Y_{k+1}(1)=\frac{Y_{k+1}(1)^{2}}{Y_{k+1}(1)} .
$$

We will show in Lemma 20 that $Y_{k+1}(1) / Y_{k}(1) \geq e^{1 / e}>1$. Thus, we can apply the same procedure as in the proof of Lemma 6 and obtain an implicit representation for the expected height:

$$
\mathbf{E} H_{n}=h_{n}+O(1),
$$

where $h_{n}=\max \left\{k: Y_{k}(1) \leq n\right\}$ and exponental bounds for the distribution of $H_{n}$ :

$$
\mathbf{P}\left\{\left|H_{n}-\mathbf{E} H_{n}\right| \geq \eta\right\} \ll e^{-\eta c}
$$

for some $c>0$.

Next we will define auxiliary functions $\bar{y}_{k}(\alpha, z)$ and $\bar{Y}_{k}(\alpha, z)$. For this purpose we have to solve an integral equation that is similar to (12). We omit the proof. It is completely the same as that of Lemma 10 . 
Lemma 18. Suppose that $1<\alpha<e^{1 / e}$ and that $\beta<e$ denotes the smallest positive solution of

$$
\alpha^{\beta}=\beta \text {. }
$$

Further, let $\mathcal{F}$ denote the set of monotonely decreasing and continuous functions $F(y)(y \geq 0)$ that satisfy

$$
\left.F(y)=1-y^{\beta}+O\left(y^{e}\right)\right) \quad(y \rightarrow 0+)
$$

and $F(y) \rightarrow 0$ as $y \rightarrow \infty$. Then there exists a unique solution $F \in \mathcal{F}$ of the integral equation

$$
F(y / \alpha)=\int_{0}^{y} F(z / \alpha) F(y-z) d z
$$

Moreover, there exists $\kappa>0$ such that

$$
F_{\alpha}(y)=O\left(e^{-y^{\kappa}}\right)
$$

as $y \rightarrow \infty$.

Again, we can assume that (after a proper scaling) $F_{\alpha}(y)=F(c y)$ satisfies $\int_{0}^{\infty} F_{\alpha}(y) d y=1$.

Next, consider the Laplace transforms

$$
\Phi_{\alpha}(u)=\int_{0}^{\infty} F_{\alpha}(y) e^{-y u} d y .
$$

They satisfy $\Phi_{\alpha}(0)=1$ and

$$
\Phi_{\alpha}^{\prime}(u)=-\frac{1}{\alpha} \Phi_{\alpha}(u) \Phi_{\alpha}(u / \alpha)
$$

As above this differential equation has a (unique entire) solution for each $\alpha>1$ (in particular for $\left.\alpha=e^{1 / e}\right)$.

For $\alpha>1$ define the auxiliary functions

$$
\bar{Y}_{k}(\alpha, z)=\alpha^{k} \Phi_{\alpha}\left(\alpha^{k}(1-z)\right),
$$

where $k$ can be considered a real (not necessarily integral) parameter and

$$
\bar{y}_{k}(\alpha, z)=\int_{0}^{z} \bar{Y}_{k}(\alpha, t) d t=\log \bar{Y}_{k+1}(\alpha, z) .
$$

The next lemma collects some facts on $\bar{Y}_{k}(\alpha, z)$ if $\alpha<e^{1 / e}$.

Lemma 19. Suppose that $1<\alpha<e^{1 / e}$ and let $\beta<e$ be given by $\alpha^{\beta}=\beta$. Let $\bar{Y}_{k}(\alpha, z)$ be defined by (26). Then we have

(1) For all $k>0$ the function $\bar{Y}_{k}(\alpha, z)$ in monotone for $z \geq 0$. The initial value satisfies $0<\bar{Y}_{k}(\alpha, 0)<1$, more precisely, $1-\bar{Y}_{k}(\alpha, 0) \sim C \alpha^{-\beta k}$ for some constant $C$ depending on $\alpha$. Furthermore we have $\bar{Y}_{k}(\alpha, 1)=\alpha^{k}$.

(2) The functions $\bar{Y}_{k}(\alpha, z)$ satisfy the recurrence relation

$$
\bar{Y}_{k+1}^{\prime}(z)=\bar{Y}_{k+1}(z) \bar{Y}_{k}(z) .
$$

(3) For all integers $\ell \geq 0$ and for all real numbers $k>0$ the difference $Y_{\ell}(z)-$ $\bar{Y}_{k}(\alpha, z)$ has exactly one positive zero $z_{\ell, k}$. In particular we have $\bar{Y}_{k}(z) \leq$ $Y_{\ell}(z)$ for $0 \leq z \leq z_{\ell, k}$ and $\bar{Y}_{k}(z) \geq Y_{\ell}(z)$ for $z \geq z_{\ell, k}$.

Note that $y_{k}(z)=\log Y_{k+1}(z)$ and $\bar{y}_{k}(\alpha, z)=\log \bar{Y}_{k+1}(\alpha, z)$. Hence, corresponding properties are also true for $\bar{y}_{k}(\alpha, z)$ and $y_{k}(z)$.

The next lemma is an analogue to Lemma 12.

Lemma 20. We have $\lim _{k \rightarrow \infty} Y_{k+1}(1) / Y_{k}(1)=e^{1 / e}$ and consequently

$$
\text { E } H_{n} \sim e \log n .
$$


Proof. Suppose that $1<\alpha<e^{1 / e}$ and set $e_{k}:=y_{k}(1) /(\log \alpha)-1$. Then the function $\bar{y}_{e_{k}}(\alpha, z)$ satisfies $\bar{y}_{e_{k}}(\alpha, 0)<y_{k}(0)$ and

$$
\bar{y}_{e_{k}}(\alpha, 1)=\tilde{y}_{k}(1) .
$$

Hence, by Lemma 19 (reformulated for $\bar{y}_{k}(\alpha, z)$ ) it follows that $\bar{y}_{e_{k}}(\alpha, z) \leq \tilde{y}_{k}(z)$ for $0 \leq z \leq 1$. Hence, by integration it also follows that $\bar{y}_{e_{k}+1}(\alpha, z) \leq \tilde{y}_{k+1}(z)$ for $0 \leq z \leq 1$. In particular,

$$
\bar{y}_{e_{k}+1}(\alpha, 1)=y_{k}(1)+\log \alpha \leq y_{k+1}(1)
$$

Thus, we have $y_{k+1}(1)-y_{k}(1) \geq \log \alpha$ for all $\alpha<e^{1 / e}$ and consequently $y_{k+1}(1)-$ $y_{k}(1) \geq 1 / e$. This also shows that $Y_{k}(1) / Y_{k-1}(1) \geq e^{1 / e}$.

In a second step we will show that for every $\varepsilon>0$

$$
y_{k}(1) \leq \frac{k}{e}(1+\varepsilon)
$$

for sufficiently $k \geq k_{0}(\varepsilon)$. Of course, this is sufficient to complete the proof of Lemma 20.

We again fix $\alpha<e^{1 / e}$ and define $t(\alpha)>0$ by

$$
(1+t(\alpha)) \alpha^{\beta} \log \alpha=1 .
$$

Note that $\lim _{\alpha \rightarrow \alpha_{0}} t(\alpha)=0$.

Further, set $\delta_{k}(z)=y_{k}(z)-\bar{y}_{k+r}(\alpha, z)$, where $r \geq 0$ is a parameter that will be chosen appropriately. Note that $\tilde{y}_{k}(z) \leq \tilde{y}(z)$ and $\bar{y}_{k+r}(\alpha, z) \leq \tilde{y}(z)$ for $0 \leq z<1$. By induction it follows that

$$
\delta_{k}(z) \leq \sum_{\ell=0}^{k} \delta_{\ell}(0) \frac{L(z)^{k-\ell}}{(k-\ell) !},
$$

where $L=\log 1 /(1-z)$. We now suppose that $r=2 k t(\alpha)-1$, set $z^{\prime}=1-\alpha^{-k(1+t(\alpha))}$ and estimate $y_{k}\left(z^{\prime}\right)=\bar{y}_{k+2 k t(\alpha)-1}\left(\alpha, z^{\prime}\right)+\delta_{k}\left(z^{\prime}\right)$ from above. We have

$$
\bar{y}_{k+2 k t(\alpha)-1}\left(\alpha, z^{\prime}\right)=k(1+t(\alpha))-\frac{C}{\alpha^{\beta k t(\alpha)}}(1+o(1))
$$

and

$$
\begin{aligned}
\delta_{k}\left(z^{\prime}\right) & \leq \sum_{\ell=0}^{k} \frac{C}{\alpha^{\beta \ell+2 \beta k t(\alpha)}} \frac{(k(1+t(\alpha)) \log \alpha)^{k-\ell}}{(k-\ell) !} \\
& =\frac{C}{\alpha^{\beta k(1+2 t(\alpha))}} \sum_{\ell=0}^{k} \frac{\left(k(1+t(\alpha)) \alpha^{\beta} \log \alpha\right)^{k-\ell}}{(k-\ell) !} \\
& =\frac{C}{\alpha^{\beta k(1+2 t(\alpha))}} \sum_{\ell=0}^{k} \frac{k^{k-\ell}}{(k-\ell) !} \\
& \sim \frac{C}{\alpha^{\beta k(1+2 t(\alpha))}} \frac{e^{k}}{2} \\
& =\frac{1}{2} \frac{C}{\alpha^{\beta k t(\alpha)}}
\end{aligned}
$$

and consequently

$$
y_{k}\left(z^{\prime}\right) \leq k(1+t(\alpha)) \log \alpha-\frac{1}{2} \frac{C}{\alpha^{\beta k t(\alpha)}}(1+o(1)) .
$$

If we compare that with

$$
\bar{y}_{k+3 k t(\alpha)-1}\left(\alpha, z^{\prime}\right)=k(1+t(\alpha)) \log \alpha-\frac{C}{\alpha^{2 \beta k t(\alpha)}}(1+o(1))
$$

we observe that (for sufficiently large $k$ )

$$
y_{k}\left(z^{\prime}\right) \leq \bar{y}_{k+3 k t(\alpha)-1}\left(\alpha, z^{\prime}\right) .
$$


Since $y_{k}(0)>\bar{y}_{k+3 k t(\alpha)}(\alpha, 0)$ it follows from Lemma 19 (resp. from its reformulation to $\left.\bar{y}_{k}(\alpha, z)\right)$ that $\tilde{y}_{k}(z) \leq \bar{y}_{k+3 k t(\alpha)}(\alpha, z)$ even for all $z \geq z^{\prime}$. In particular we have (for sufficiently large $k$ )

$$
y_{k}(1) \leq \bar{y}_{k+3 k t(\alpha)-1}(\alpha, 1)=k(1+3 t(\alpha)) \log \alpha \leq \frac{k}{e}(1+3 t(\alpha))
$$

Since we can choose $\alpha$ that $t(\alpha)$ is arbitrarily small this completes the proof of the lemma.

The remaining parts of the proof of Theorem 3 is completely analogous to the corresponding part of the proof of Theorem 2. One has to show that (24) has a unique solution for $\alpha_{0}=e^{1 / e}$ and that $\bar{Y}_{e_{k}}\left(e^{1 / e}, z\right)$ is a proper approximation for $Y_{k}(z)$. This leads to an approximation for $\mathbf{P}\left\{H_{n} \leq k\right\}$ of the form $F_{e^{1 / e}}\left(n / Y_{k}(1)\right)$. We leave the details to the reader.

\section{SCALE-FrEe TREeS}

Plane oriented trees are defined by $\Psi(w)=1 /(1-w)$ and do not constitute a polynomial increasing tree family, either, but their behaviour is also very similar to $d$-ary increasing trees. In fact, we will deal with generalized plane oriented trees defined by $\Psi(w)=1 /(1-w)^{r}$, where $r$ is a positive rational number. These kinds of trees are also called scale-free trees (see [11]) because the tail of the degree distribution has an asymptotic power law. They are also constructed by a tree evolution process, where the probability that a node of out-degree $j$ is chosen is proportial to $r+j$ (compare with Section 3). Unfortunately we are only able to discuss the irrational case in full generatlity. (Exponential tail estimates the heigth distribution can be obtained for all cases, see [10].)

Recall that $y(z)=\sum_{n \geq 1} y_{n} z^{n} / n$ ! that satisfies $y^{\prime}(z)=(1-y(z))^{-r}$ is given by

$$
y(z)=1-(1-(r+1) z)^{1 /(r+1)}
$$

and the coefficients by

$$
y_{n}=n !(-1)^{n-1}(r+1)^{n}\left(\begin{array}{c}
1 /(r+1) \\
n
\end{array}\right) .
$$

The height distribution for scale-free trees is encoded by the generating functions $y_{k}(z)=\sum_{n \geq 0} y_{n} \mathbf{P}\left\{H_{n} \leq k\right\} z^{n} / n$ ! that are given by $y_{0}(z)=0$ and recusively by

$$
y_{k+1}^{\prime}(z)=\frac{1}{\left(1-y_{k}(z)\right)^{r}} \quad\left(y_{k+1}(0)=0\right) .
$$

By taking derivatives it follows that

$$
y_{k+1}^{\prime \prime}(z)=r\left(y_{k+1}^{\prime}(z)\right)^{1+\frac{1}{r}} y_{k}^{\prime}(z) .
$$

Let $r=A / B$ where $A, B$ are coprime positive integers and set

$$
Y_{k}(z)=\left(y_{k}^{\prime}(z)\right)^{\frac{1}{A}} .
$$

Here we have $Y_{1}(z)=1$ and

$$
Y_{k+1}^{\prime}(z)=\frac{1}{B} Y_{k+1}(z)^{B+1} Y_{k}(z)^{A} \quad\left(Y_{k+1}(0)=1\right)
$$

Thus, $Y_{k}(z)$ looks very similar to $\bar{y}_{k}(z)$ in the case of $(A+B+1)$-ary increasing trees. However, $Y_{k}(z)$ does not directly encode the height distribution. In particular, we have to use a two step procudure. First we work with $Y_{k}(z)$ and then we apply the results for

$$
y_{k}^{\prime}(z)=Y_{k}(z)^{A}=\sum_{n \geq 0} y_{n+1} \mathbf{P}\left\{H_{n+1} \leq k\right\} \frac{z^{n}}{n !} .
$$


We start with an analogue to Lemma 5 resp. Lemma 16 which is in fact a key property for the whole proof of Theorem 4. The proof of Lemma 21 is completely the same as that of Lemma 16.

Lemma 21. Suppose that $Y_{1}(z), Y_{2}(z), \bar{Y}_{1}(z), \bar{Y}_{2}(z)$ are non-negative continuous functions that are defined for $z \geq 0$ such that $Y_{1}(0)<\bar{Y}_{1}(0), Y_{2}(0)<\bar{Y}_{2}(0)$, $Y_{2}^{\prime}(z)=\frac{1}{B} Y_{2}(z)^{B+1} Y_{1}(z)^{A}, \bar{Y}_{2}^{\prime}(z)=\frac{1}{B} \bar{Y}_{2}(z)^{B+1} \bar{Y}_{1}(z)^{A}$, and that the difference $\bar{Y}_{1}(z)-Y_{1}(z)$ has exactly one positive zero. Then the difference $\bar{Y}_{2}(z)-Y_{2}(z)$ has at most one positive zero.

Next one can prove is an analogue to Lemma 17:

$$
\frac{Y_{k+2}(1 /(r+1))}{Y_{k+1}(1 /(r+1))} \leq \frac{Y_{k+1}(1 /(r+1))}{Y_{k}(1 /(r+1))}
$$

The proof is a mixture of the proofs of Lemma 9 and Lemma 17 (see also [10]).

Thus, the limit $\lim _{k \rightarrow \infty} Y_{k+1}(1 /(r+1)) / Y_{k}(1 /(r+1))$ exists. If we know that this limit is greater than 1 then it also follows that $\lim _{k \rightarrow \infty} y_{k+1}^{\prime}(1 /(r+1)) / y_{k}^{\prime}(1 /(r+1))>1$. In that case we can proceed similarly to Lemma 6 and obtain an implicit representation for the expected height:

$$
\mathbf{E} H_{n}=h_{n}+O(1) \text {, }
$$

where $h_{n}=\max \left\{k: y_{k}^{\prime}(1 /(r+1))^{1+\frac{1}{r}} \leq n\right\}$ and exponentail bounds for the distribution of $H_{n}$ :

$$
\mathbf{P}\left\{\left|H_{n}-\mathbf{E} H_{n}\right| \geq \eta\right\} \ll e^{-\eta c}
$$

for some $c>0$. This proves the concentration property of Theorem 4 .

We now show that we actually have

$$
\lim _{k \rightarrow \infty} Y_{k+1}(1 /(r+1)) / Y_{k}(1 /(r+1))>1 .
$$

For this purpose we use (again) proper solutions of the integral equation

$$
\begin{aligned}
y^{\frac{1}{A+B}} F(y / \alpha)=\frac{\Gamma\left(1+\frac{1}{A+B}\right)}{\Gamma\left(\frac{1}{A+B}\right)^{A+B+1}} \int_{y_{1}+\cdots+y_{A+B+1}=y, y_{j} \geq 0} & \prod_{j=1}^{B+1}\left(F\left(y_{j} / \alpha\right) y_{j}^{\frac{1}{A+B}-1}\right) \\
& \times \prod_{\ell=B+2}^{A+B+1}\left(F\left(y_{\ell}\right) y_{\ell}^{\frac{1}{A+B}-1}\right) d \mathbf{y} .
\end{aligned}
$$

If $1<\alpha<\alpha_{0}$ then there exists a unique solution of the form $F_{\alpha}(y)=1-c y^{\beta}+O\left(y^{\gamma}\right)$ $(y \rightarrow 0+)$, where $\beta$ is the smallest positive solution of the equation $(B+1)+A \alpha^{\beta}=$ $1+(A+B) \beta$ or of

$$
r \alpha^{\beta}+1=(r+1) \beta .
$$

The constant $c>0$ is chosen in a way that

$$
\int_{0}^{\infty} F_{\alpha}(y) y^{\frac{1}{A+B}-1} e^{-u y} d y=(r+1)^{\frac{1}{A+B}} \Gamma\left(\frac{1}{A+B}\right) .
$$

Furthermore, we have $F(y)=O\left(e^{-C y^{\kappa}}\right)$ for some constants $C>0$ and $\kappa>0$.

Withe help of $F(y)$ we define

$$
\Phi_{\alpha}(u)=\frac{1}{(r+1)^{\frac{1}{A+B}} \Gamma\left(\frac{1}{A+B}\right)} \int_{0}^{\infty} F_{\alpha}(y) y^{\frac{1}{A+B}-1} e^{-u y} d y .
$$

These are entire functions and are also given by $\Phi_{\alpha}(0)=1$ and by the differential equation

$$
\Phi_{\alpha}^{\prime}(u)=-\frac{1}{B} \frac{1}{\alpha^{A /(A+B)}} \Phi_{\alpha}(u)^{B+1} \Phi_{\alpha}\left(\frac{u}{\alpha}\right)^{A} .
$$


Finally define auxiliary functions

$$
\bar{Y}_{k}(\alpha, z)=\alpha^{k /(A+B)} \Phi_{\alpha}\left(\alpha^{k}(\rho-z)\right),
$$

where $\rho=1 /(r+1)$. These functions satisfy

$$
\bar{Y}_{k+1}^{\prime}(\alpha, z)=\frac{1}{B} \bar{Y}_{k+1}(\alpha, z)^{B+1} \bar{Y}_{k}(\alpha, z)^{A},
$$

$0<\bar{Y}_{k}(\alpha, 0)<1$ (more precisely $\left.1-\bar{Y}_{k}(\alpha, 0) \sim C \alpha^{-\beta k}\right)$ and $\bar{Y}_{k}(\alpha, 1 /(r+1))=$ $\alpha^{k /(A+B)}$

By using the same ideas as in the first part of the proof of Lemma 12 it follows that $Y_{k+1}(1 /(r+1)) / Y_{k}(1 /(r+1)) \geq \alpha^{1 /(A+B)}>1$ for all $\alpha<\alpha_{0}$. In particular, this completes the proof of (28) and consequently the concentration property of $H_{n}$.

Furthermore, these auxiliary functions can be also used to determine the limit of $Y_{k+1}(1 /(r+1)) / Y_{k}(1 /(r+1))$ (and consequently we get an asymptotic representation of $\mathbf{E} H_{n}$, see Lemma 22$)$.

Next set

$$
G_{\alpha}(y)=\frac{\Gamma\left(\frac{A}{A+B}\right)}{\Gamma\left(\frac{1}{A+B}\right)^{A}} \int_{z_{1}+\cdots+z_{A}=1, z_{j} \geq 0} \prod_{j=1}^{A}\left(F\left(y z_{j}\right) z_{j}^{\frac{1}{A+B}-1}\right) d \mathbf{z}
$$

and

$$
\bar{\Phi}_{\alpha}(u)=\Phi_{\alpha}(u)^{A}=\frac{1}{(r+1)^{\frac{r}{1+r}} \Gamma\left(\frac{r}{1+r}\right)} \int_{0}^{\infty} G(y) y^{-\frac{1}{1+r}} e^{-y u} d y .
$$

Alternatively, $\bar{\Phi}_{\alpha}(u)$ is given by $\bar{\Phi}_{\alpha}(0)=1$ and by the differential equation

$$
\bar{\Phi}_{\alpha}^{\prime}(u)=-\frac{r}{\alpha^{r /(1+r)}} \bar{\Phi}_{\alpha}(u)^{1+\frac{1}{r}} \bar{\Phi}_{\alpha}\left(\frac{u}{\alpha}\right) .
$$

Hence, the functions

$$
\bar{y}_{k}^{\prime}(\alpha, z)=\alpha^{\frac{r k}{1+r}} \bar{\Phi}\left(\alpha^{k}(\rho-z)\right)
$$

satisfy

$$
\bar{y}_{k+1}^{\prime \prime}(\alpha, z)=r\left(\bar{y}_{k+1}^{\prime}(\alpha, z)\right)^{1+\frac{1}{r}} \bar{y}_{k}^{\prime}(\alpha, z),
$$

$0<\bar{y}_{k}^{\prime}(\alpha, 0)<1$ (more precisely $\left.1-\bar{y}_{k}^{\prime}(\alpha, 0) \sim C^{\prime} \alpha^{-\beta k}\right)$ and $\bar{y}_{k}^{\prime}(\alpha, 1 /(r+1))=$ $\alpha^{r k /(1+r)}$

Finally, if we consider

$$
\bar{y}_{k}(\alpha, z)=\int_{0}^{z} \bar{y}_{k}^{\prime}(\alpha, t) d t
$$

then we have

$$
\bar{y}_{k+1}^{\prime}(\alpha, z)=\frac{1}{\left(1-\bar{y}_{k}(\alpha, z)\right)^{r}}
$$

with $\bar{y}_{k}(\alpha, 0) \sim-C^{\prime \prime} \alpha^{-\beta k}$ and $1-\bar{y}_{k}(\alpha, 1 /(r+1)) \sim \alpha^{-(k+1) /(r+1)}$.

Now we are in a position to prove the next property.

Lemma 22. We have $\lim _{k \rightarrow \infty} y_{k+1}^{\prime}(1 /(r+1)) / y_{k}^{\prime}(1 /(r+1))=e^{1 /\left(c_{r}\left(1+\frac{1}{r}\right)\right)}$ and consequently

$$
\mathbf{E} H_{n} \sim c_{r} \log n
$$

Proof. As already mentioned it follows as in the proof of Lemma 12 that $Y_{k+1}(\rho) / Y_{k}(\rho) \geq \alpha^{1 /(A+B)}>1$ for all $\alpha<\alpha_{0}$. Consequently, $y_{k+1}^{\prime}(\rho) / y_{k}^{\prime}(\rho) \geq$ $\alpha_{0}^{1 /\left(1+\frac{1}{r}\right)}$. (Here $\rho=1 /(r+1)$.)

In order to prove a corresponding upper bound it is sufficient to prove that

$$
y_{k}(\rho) \leq 1-\alpha_{0}^{\frac{k}{1+r}(1+\varepsilon)}
$$


for sufficiently large $k$. We again fix $\alpha<\alpha_{0}$ and define $t(\alpha)>0$ by

$$
(1+t(\alpha)) \alpha^{\beta} \log \alpha=1+\frac{1}{r} .
$$

Note that $\lim _{\alpha \rightarrow \alpha_{0}} t(\alpha)=0$.

If $\delta_{k}(z)=y_{k}(z)-\bar{y}_{k+r}(\alpha, z)$ then we have

$$
\delta_{k}(z) \leq \sum_{\ell=0}^{k} \delta_{\ell}(0) \frac{\left(\frac{r}{r+1} L(z)\right)^{k-\ell}}{(k-\ell) !},
$$

where $L=\log 1 /(1-(r+1) z)$. As in the proof of Lemma 12 we set $r=2 k t(\alpha)$ and $z^{\prime}=\frac{1}{r+1}-\alpha^{-k(1+t(\alpha))}$ and estimate $\tilde{y}_{k}\left(z^{\prime}\right)=\bar{y}_{k+2 k t(\alpha)}\left(\alpha, z^{\prime}\right)+\delta_{k}\left(z^{\prime}\right)$ from above. We leave the details to the reader.

The remaining parts of the proof of Theorem 4 are (again) completely analogous to the corresponding parts of the proof of Theorem 2 resp. of Theorem 3. One has to show that (29) has a unique solution for $\alpha_{0}=e^{1 / c_{r}^{\prime}}$ and that $\bar{y}_{e_{k}}^{\prime}\left(e^{1 / c_{r}^{\prime}}, z\right)$ is a proper approximation for $y_{k}(z)$. This leads to an approximation for $\mathbf{P}\left\{H_{n} \leq k\right\}$ of the form $G_{e^{1 / c_{r}^{\prime}}}\left((r+1) n /\left(y_{k}^{\prime}(\rho)\right)^{1+\frac{1}{r}}\right)$. We leave (again) the details to the reader.

\section{REFERENCES}

[1] F. Bergeron, P. Flajolet, And B. Salvy, Varieties of increasing trees, CAAP '92 (Rennes, 1992), 24-48, Lecture Notes in Comput. Sci., 581, Springer, Berlin, 1992.

[2] N. Broutin, L. Devroye, E. Mcleish and M. De la Salle, The height of increasing trees, manuscript, 2006.

[3] B Chauvin and M. Drmota, The Random Bisection Problem, Travelling Waves, and the Distribution of the Height of Binary Search Trees, Algorithmica, submitted.

[4] L. Devroye, A note on the height of binary search trees, J. Assoc. Comput. Mach. 33 (1986), $489-498$.

[5] L. Devroye, Branching processes in the analysis of the height of trees, Acta Inform. 24 (1987), 277-298.

[6] L. Devroye And B. Reed, On the variance of the height of random binary search trees, SIAM J. Comput. 24 (1995), 1157-1162.

[7] M. Drmota, The Saturation Level in Binary Search Trees, in: Mathematics and Computer Science. Algorithms, Trees, Combinatorics and Probabilities (D. Gardy, A. Mokkadem eds.), Birkhäuser, Basel, 2000, pp. 41-51.

[8] M. Drmota, An Analytic Approach to the Height of Binary Search Trees, Algorithmica 29, 89-119, 2001.

[9] M. Drmota, An Analytic Approach to the Height of Binary Search Trees. II, J. Assoc. Comput. Mach. 50 (2003), 333-374.

[10] M. Drmota, Concentration Properties of Extremal Parameters in Random Discrete Structures, Discr. Math. Theor. Comput. Sci., proc. AG, 1-30, 2006

[11] Z. Katona, Width of a scale-free tree, J. Appl. Probab. 42 (2005), 839-850.

[12] A. Panholzer and H. Prodinger, The level of nodes in increasing trees revisited, Random Struct. Algorithms, to appear.

[13] B. Pittel, Note on the height of recursive trees and $m$-ary search trees, Random Struct. Algorithms 5 (1994), 337-347.

[14] B. ReED, The height of a random binary search tree, J. Assoc. Comput. Mach., 50 (2003), 306-332.

[15] R. T. Smythe and H. M. Mahmoud, A survey of recursive trees, Theory Probab. Math. Statist. 51 (1995), 1-27. 